\title{
Investigation of Topographic Site Effects using 3D Waveform Modelling: Amplification, Polarization and Torsional Motions in the Case Study of Arquata del Tronto (Italy)
}

\section{Julie Baron}

Istituto Nazionale di Oceanografia e Geofisica Sperimentale

llaria Primofiore

Ministero dell'Istruzione, dell'Università e della Ricerca

\section{Peter Klin ( $\nabla$ pklin@inogs.it )}

Istituto Nazionale di Oceanografia e Geofisica Sperimentale https://orcid.org/0000-0002-5373-4484

\section{Giovanna Laurenzano}

Istituto Nazionale di Oceanografia e Geofisica Sperimentale

\section{Giovanna Vessia}

Universita degli Studi Gabriele d'Annunzio Chieti Pescara

\section{Research Article}

Keywords: Topographic amplification, directional site effect, torsional motion, numerical modeling, Arquata del Tronto, SPECFEM3D, cross-coupling of motion components in site response

Posted Date: June 4th, 2021

DOl: https://doi.org/10.21203/rs.3.rs-582452/v1

License: (c) (i) This work is licensed under a Creative Commons Attribution 4.0 International License. Read Full License

Version of Record: A version of this preprint was published at Bulletin of Earthquake Engineering on December 3rd, 2021. See the published version at https://doi.org/10.1007/s10518-021-01270-2. 
2 Investigation of topographic site effects using 3D

3 waveform modelling: amplification, polarization and

4 torsional motions in the case study of Arquata del Tronto

5 (Italy)

6

7 Julie Baron ${ }^{1}$, Ilaria Primofiore ${ }^{2}$, Peter Klin $^{1 *}$, Giovanna Vessia ${ }^{2}$, Giovanna Laurenzano ${ }^{1}$

8

9 ' National Institute of Oceanography and Applied Geophysics - OGS, Borgo Grotta Gigante 42/C, 34010 Sgonico,

10 Trieste, Italy

$11{ }^{2}$ University "G. d' Annunzio" of Chieti-Pescara, Department of Engineering and Geology, Via dei Vestini 31,

1266100 Chieti, Italy

13 * Corresponding author. E-mail address: pklin@inogs.it

Julie Baron https://orcid.org/0000-0002-3215-6996

Ilaria Primofiore https://orcid.org/0000-0002-5688-8067

Peter Klin https://orcid.org/0000-0002-5373-4484

Giovanna Vessia https://orcid.org/0000-0003-1733-7112

Giovanna Laurenzano https://orcid.org/0000-0002-2618-3007 
The combined effect of topography and near-surface heterogeneities on the seismic response is hardly predictable and may lead to an aggravation of the ground motion. We apply physics-based numerical simulations of 3D seismic wave propagation to highlight these effects in the case study of Arquata del Tronto, a hamlet in the Apennines that suffered irregularly distributed damage during the 2016 seismic sequence in Central Italy. We analyze the linear visco-elastic seismic response for vertically incident plane waves in terms of spectral amplification, polarization and induced torsional motion within the frequency band 1-8 $\mathrm{Hz}$ over a 1 $\mathrm{km}^{2}$ square area, with spatial resolution $25 \mathrm{~m}$. To discern the effects of topography from those of the sub-surface structure we iterate the numerical simulations for three different versions of the structural model: one homogeneous, one with a surficial weathering layer and a soil basin and one with a complex internal structure. The numerical results confirm the correlation between topographic curvature and amplification and support a correlation between the induced torsional motion and the topographic slope. On the other hand we find that polarization does not necessarily imply ground motion amplification. In the frequency band above $4 \mathrm{~Hz}$ the topography-related effects are mainly aggravated by the presence of the weathering layer, even though they do not exceed the soil-related effects in the flat-topography basin. The structure below the weathering layer plays a recognizable role in the topography-related site response only for frequencies below $4 \mathrm{~Hz}$.

Topographic amplification, directional site effect, torsional motion, numerical modeling, Arquata del Tronto, SPECFEM3D, cross-coupling of motion components in site response

\section{Introduction}

The estimation of ground motion amplification due to site effects plays a fundamental role in the efforts devoted to seismic-risk mitigation (Amanti et al. 2020). Thanks to ground motion recordings and earthquake damage reports from sites characterized by topography, it has long been known that topographic features are an indicator of site effects, but theoretical explanations based on the relief's geometrical characteristics usually underestimated the observed amplification values (Geli et al. 1988). The discrepancies between predictions and observations can be ascribed to the absence of adequate reference site in many experimental estimates (Pedersen et al. 1994; Wood and Cox 2016) on one hand, and on the other hand, to the adoption of oversimplified models in theoretical estimates. The oversimplification that may cause underestimation of theoretically predicted amplifications typically consists of inadequate two-dimensional (2D) approximation of prominently three-dimensional (3D) topographic features (Paolucci 2002; Luo et al. 2020) or in neglecting impedance contrasts in the subsurface structure that possibly concur with topography in forming amplification effects (Graizer 2009; Assimaki and Jeong 2013; Hailemikael et al. 2016, to cite a few).

There is today a general agreement that topography implies a number of effects, discussed in the overview provided by Massa et al. (2014) and briefly summarized in the points below:

- Amplification occurs at the top of the topographic high in the wavelength range comparable to the width of the morphological relief (peak frequency decreases with increasing relief width).

- Amplification is proportional to the shape ratio (height vs. base half-width) of the relief.

- Amplification is larger for horizontal components, in particular for the component transverse to the direction of elongation of the relief.

- Topographic amplification is dependent on the source-to-site direction, numerical results however indicate that it is maximized for waves with vertical incidence.

- Topographic effects combined with the effects of structural heterogeneities, give rise to atypical topographic effects.

In order to account for possible topography-related amplification effects, a number of international seismic codes (e.g. Eurocode 8, NTC18 in Italy) have introduced the topographic aggravation factor (TAF) based on approximate topographic descriptions. Recently the efforts are focused on the development of more sophisticated TAF estimators, based on the availability of digital elevation maps (DEM) and equivalent uniform rock stiffness estimates (Grelle et al. 2018). Zhou et al. (2020) adopt the back-propagation neural network technique for the derivation of a ground motion amplification model based on topographic geometrical features estimated from the DEM. Since they are based on surface topography alone, these approaches neglect possible interactions between topographic and structural effects on wave propagation. According to Burjánek et al. (2014), the observed amplification at sites with comparable topography but with different subsurface structure may differ for a factor which is significantly larger than the expected ground motion variability and studies based exclusively on the terrain topography have almost no chance to capture the site effects correctly. 
In the present work we address the problem of possible ground motion aggravation due to the complex interaction between a pronounced topographic relief and a heterogeneous distribution of underground seismic velocities. Given that the distinction of topographic effects from the stratigraphic ones in the seismic records results impractical, we adopted an approach that fully relies on numerical simulations to separate the two (Ashford et al. 1997). Three-dimensional (3D) physics-based numerical simulations of seismic wave propagation can provide a detailed and accurate characterization of the seismic ground motion and allow us to explore its spatial heterogeneity. This kind of numerical simulations have proven to be essential for the explanation of anomalies in the observed ground motion during past earthquakes (e. g. Paolucci et al. 2015; Cruz-Atienza et al. 2016; Klin et al. 2019), also in cases in which topographic effects are involved (e.g. Puglia et al. 2013; Luo et al. 2020). Moreover, numerical simulations allow the quantification of potential amplification effects at sites where ground motion data is lacking (e. g. Fehr et al. 2019).

In the present work we analyze the site response not only in the conventional terms of spectral amplification but also in terms of induced polarization and induced rotations in the horizontal components of ground motion. According to several works (e. g. Burjánek et al. 2014; Massa et al. 2014; Pischiutta et al. 2018), the polarization of the site response can be viewed as an indicator of topography-induced strong site effects, even though the direction of polarization is not strictly related with the main directions of the topographic features. Given that the polarization of the site response can be identified by low-cost single-station analyses, deepening this topic could provide useful indications for micro-zonation studies.

On the other hand, ground motion rotations are rarely considered in site effects studies, due to difficulties involved in measuring rotational motions and strains and because of a widespread preconception in the seismological community that rotational motions are insignificant (Lee et al. 2009). In the earthquake engineering community instead, the ground rotational motion gained interest (Lee and Trifunac 1985; Castellani et al. 2012), since the travelling wave effect was recognized by Newmark (1969) and it is today accounted for in building codes under the term accidental eccentricity (Anagnostopoulos et al. 2015). While the research regarding the relevance of ground motion rotations is still undergoing (Guidotti et al. 2018), in the present work we investigate the possibility of topography-induced rotations around the vertical axis (torsional motion). Topography-related torsional motions were observed - without a quantitative analysis - by Stupazzini et al. (2009) in their 3D numerical simulations of earthquake ground motion in the Grenoble valley. In the present work we set up a simple predictive model which relates torsional motions and the topographic slope in homogeneous media and we evaluate the possible variations due to near surface heterogeneities. We elected as case study for the aforementioned investigations the $1 \mathrm{~km}^{2}$ square area centered in the historical center of Arquata del Tronto, a village in the Central Apennines located on an elongated WNW-ESE ridge at an elevation of about 100 meters above the underlying valley. The village suffered extensive damage and collapses in the 2016 August $24^{\text {th }} \mathrm{Mw}$ 6.0 Amatrice earthquake and the non-uniform damage distribution indicated possible topography-related site effects (Galli et al. 2016; Lanzo et al. 2019). An evaluation of site effects in Arquata del Tronto and Borgo from seismological data has been performed by Laurenzano et al. (2019), who exploited earthquake recordings acquired on temporary seismic stations deployed after the first mainshock of the 2016 seismic sequence. Their study evidenced a substantial difference between the frequency-dependent site amplification function at a location on the top of the ridge and in the valley below. A directional effect that could be associated to the ridge orientation has been pointed out, as well. In order to quantitatively explain the observed site response characteristics, Giallini et al. (2020) applied 2D numerical simulations using subsoil models deduced from the available geognostic, geophysical and geomechanical surveys and evidenced the coupling effect of stratigraphy and topography associated with the presence of a weathered portion of the rock mass and the alternation of highly dipping rocky materials. They admitted that $2 \mathrm{D}$ analyses cannot fully capture the behavior of such an asymmetric ridge and suggested for this site tridimensional numerical analyses. This origin of the site effects was finally verified by Primofiore et al. (2020), who set up an updated 3D digital model of the area and evaluated the resulting ground motion by means of 3D numerical simulations of seismic wave propagation.

The present work can be therefore considered as a follow up of the analyses by Primofiore et al. (2020). In order to provide general results and some new insights with respect to the topography-related site effects, we iterate the numerical evaluation of ground motion for three different versions of the Arquata del Tronto visco-elastic structural model, with growing complexity. The first version is characterized by a homogeneous structure, the second one presents near surface alterations (weathering layer and a restricted soil basin over a flat area), and the third one exhibits a complex internal structure and corresponds to the one used in Primofiore et al. (2020). The differences between the seismic responses evaluated for each model are then measured in terms of the effect size in three different frequency bands of engineering interest. We also analyze the possible correlation between the amplification and the topographic curvature, which was proposed as a proxy for topographic amplification by Maufroy et al. (2015). 


\section{Description of site response in terms of response function $3 \times 3$ matrix}

In our characterization of site effects we follow Paolucci (1999) and employ 3D impulse response functions evaluated from 3D physics-based numerical simulations of seismic wave propagation. The 3D response function consists of a $3 \times 3$ matrix with components $h_{i j}(\boldsymbol{x}, t)$. The time series $h_{i j}(\boldsymbol{x}, t)$ describes the $i$-th spatial component of the seismic time series that is observed at the site $\boldsymbol{x}$ when the area is invested by a vertically incident impulsive plane wave polarized in the $j$-th direction with unitary amplitude. We can express the ground motion $\boldsymbol{y}(\boldsymbol{x}, t)=\left[y_{l}(\boldsymbol{x}, t), y_{2}(\boldsymbol{x}, t), y_{3}(\boldsymbol{x}, t)\right]$ at the site $\boldsymbol{x}$ for any vertically incident seismic input $\boldsymbol{u}(t)=\left[u_{1}(t), u_{2}(t), u_{3}(t)\right]$ as

$$
y_{i}(x, t)=\sum_{j} h_{i j}(x, t) * u_{j}(t)
$$

where $*$ denotes convolution in time. In virtue of the convolution theorem, the expression can be evaluated as a multiplication in the frequency domain

$$
Y_{i}(x, f)=\sum_{j} H_{i j}(x, f) U_{j}(f)
$$

where we denote with uppercase letters the Fourier spectrum of the corresponding time-domain quantities denoted in lowercase letters.

If an ideal elastic medium was considered, we could numerically evaluate the terms $h_{i j}(\boldsymbol{x}, t)$ as the three component solutions of the wave field at the topographic surface for three cases of unitary impulsive plane wave entering the spatial domain from below. In the present case however, a viscoelastic medium is considered and the intrinsic attenuation (and dispersion) would imply a response function which is dependent on the depth at which the plane wave is excited. In order to get rid of this incongruence, we adopted a redefinition of 3D response functions based on the concept of the reference site. In this approach, we evaluate the solutions for polarized plane waves also in a "reference" spatial domain, characterized by a homogeneous medium (having the properties of the bedrock in the geomodel under investigation) and flat topographic surface (with elevation equal to the average of the studied case). The depth at which the plane wave is introduced is irrelevant, as long as it is the same in the two cases. If we denote the frequency domain solutions in the reference medium and in the investigated medium with $U_{i j}^{r e f}$ and $U_{i j}$ respectively, (with the index $i$ denoting the spatial component of the solution and the index $j$ denoting the polarization of the excited plane wave) we can estimate the components of the response function of the geomodel under investigation in frequency domain as the quotients

$$
\begin{array}{lll}
H_{11} & =\frac{U_{11}}{U_{11}^{\text {ref }}} ; \quad H_{12}=\frac{U_{12}}{U_{22}^{\text {ref }}} ; \quad H_{13}=\frac{U_{13}}{U_{33}^{\text {ref }}} ; \\
H_{21} & =\frac{U_{21}}{U_{11}^{\text {ref }}} ; \quad H_{22}=\frac{U_{22}}{U_{22}^{\text {ref }}} ; \quad H_{11}=\frac{U_{23}}{U_{33}^{\text {ref }}} ; \\
H_{31} & =\frac{U_{31}}{U_{11}^{\text {ref }}} ; \quad H_{32}=\frac{U_{32}}{U_{22}^{\text {ref }}} ; \quad H_{11}=\frac{U_{33}}{U_{33}^{\text {ref }}} .
\end{array}
$$

This definition of the response functions also relaxes the request of the unitary and impulsive character of the plane wave entering the spatial domain and permits the adoption of a time function which suits at best the numerical method we use in the simulations.

\section{Computation of response function from 3D model with SPECFEM3D}

Fundamental factors for a successful numerical simulation consist in the availability of a plausible 3D structural model of the spatial domain with an adequate geophysical characterization of the geological formations and in the accurate application of a numerical method for the solution of the elastodynamic wave equation. In the present work, we constructed a digital 3D structural model of the studied area by means of the commercial software Geomodeller3D, which interpolates the available geological data with methods based on the compliance of stratigraphic rules and on the interpretation of geologic surfaces as isopotential surfaces of a scalar potential field (Calcagno et al. 2008). We considered the geological and geophysical data from the surveys performed in the area during microzonation studies (ISPRA 2018; Puzzilli et al. 2019). Details on the 3D model we built are discussed in section Models. Considering the need to solve accurately the wave propagation in a spatial domain bounded by an irregularly shaped free surface, we decided to perform the numerical simulations with the Spectral Element Method (SEM). 
This approach, first introduced in the study of acoustic and elastic waves by Seriani and Priolo (1994), combines the geometrical flexibility of Finite Elements Methods (FEM) with the accuracy of the spectral methods. In order to perform the simulations presented in the present work, we relied on the software SPECFEM3D Cartesian from source (Peter et al. 2011). The code accounts for the intrinsic attenuation properties of the viscoelastic medium by means of a series of standard linear solid elements (Savage et al. 2010) and avoids the boundary reflection artefacts thanks to the usage of the perfectly matched layer technique (Xie et al. 2014).

\section{Reduction of the $3 \times 3$ response function to the plane wave amplification function}

In seismic hazard studies the site effects are typically described as the ratio between the Fourier Amplitude Spectrum (FAS) $\left|Y_{\text {hor }}(\boldsymbol{x}, f)\right|$ of the combined horizontal components of the ground motion at the observation site $\boldsymbol{x}$ and that of the seismic input $\left|\mathrm{U}_{\text {hor }}(f)\right|$

$$
A(x, f)=\frac{\left|Y_{\text {hor }}(x, f)\right|}{\left|U_{\text {hor }}(f)\right|}
$$

where, having assumed that components 1 and 2 lay in the horizontal plane, we put

$$
\left|Y_{\text {hor }}(x, f)\right|=\frac{1}{\sqrt{2}} \sqrt{\left|Y_{1}(x, f)\right|^{2}+\left|Y_{2}(x, f)\right|^{2}}
$$

and an analogous expression for $\left|\mathrm{U}_{h o r}(f)\right|$.

According to equation (2), the horizontal components of the ground motion at the observation site depend also on the vertical components of the seismic input, which are not accounted for in the denominator of the spectral ratio defined in equation (4). Since the vertical component of the seismic input is not uniquely determined by the horizontal components, in our construction the amplification $A(\boldsymbol{x}, f)$ behaves as a random variable. Since we can expect a log-normal distribution for the spectral amplitude ratios $A_{n}(\boldsymbol{x}, f)$ corresponding to different seismic inputs denoted by the index $n$, the most efficient choice for their characterization are the geometric average $\bar{A}^{*}(x, f)$ and the multiplicative or geometric standard deviation (GSD) $\sigma^{*}(x, f)$ defined as

$$
\bar{A}^{*}(x, f)=\exp \left(\bar{A}_{l n}\right) \text { where } \bar{A}_{l n}=\frac{1}{N} \sum_{n=1}^{N} \ln \left(A_{n}(x, f)\right)
$$

$$
\sigma^{*}(x, f)=\exp \left(\sigma_{l n}\right) \text { where } \sigma_{l n}=\left(\frac{1}{N-1} \sum_{n=1}^{N}\left(\ln \left(A_{n}(x, f)\right)-\bar{A}_{l n}\right)^{2}\right)^{1 / 2} \text {. }
$$

In this study we estimate the site average amplification and its multiplicative standard deviation by considering a set of three component earthquake ground motion records as seismic inputs $\boldsymbol{U}^{(n)}(f)$ and by evaluating the correspondent theoretical site recordings $\boldsymbol{Y}^{(n)}(x, f)$ by applying eq. (2). Since we consider the numerical solutions for vertically emerging plane waves, we call this estimation of the site amplification as plane-wave amplification. The reduction of the $3 \times 3$ response function to the site amplification function can be therefore obtained by replacing in equation (6) the terms

$$
A_{n}=\sqrt{\frac{\left|H_{11} U_{1}^{(n)}+H_{12} U_{2}^{(n)}+H_{13} U_{3}^{(n)}\right|^{2}+\left|H_{21} U_{1}^{(n)}+H_{22} U_{2}^{(n)}+H_{23} U_{3}^{(n)}\right|^{2}}{\left|U_{1}^{(n)}\right|^{2}+\left|U_{2}^{(n)}\right|^{2}}}
$$

where $H_{i j}$ are 3D response functions as defined in equation (3) and where we omit the frequency and space dependence from the notation for the sake of simplicity.

In the present analysis we considered as seismic inputs the ground motion records of 200 events with magnitude in the range from $M=2.8$ to $M=4.8$ that occurred during the 2016-17 Amatrice seismic sequence (see Figs. S1 and S2 in supplemental information) and that were collected at the reference site station MZ75 on geological bedrock at Montegallo municipality, $9 \mathrm{~km}$ north of Arquata del Tronto (Laurenzano et al. 2019). The GSD considered in the results therefore depicts the amplification function's uncertainty, that is due to the variable contribution of the input motion's vertical component to the output motion's horizontal components through the cross-coupling effects (Paolucci 1999).

\section{Horizontal polarization analysis from the $3 \times 3$ response function}

Site effects on pronounced reliefs are usually correlated with ground motion directionality (Burjánek et al. 2014; Massa et al. 2014) and a polarization analysis of the site response is therefore appropriate. We limit our polarization analysis to the horizontal components and following the scheme of the plane wave amplification 
analysis we define the plane wave directional amplification $\bar{A}_{\varphi}^{*}(x, f)$ as the geometric average of the ratio of the FAS of the ground motion component in the direction $\varphi$ measured clockwise from the 2nd axis (North) and the FAS of the seismic input in the same direction

$$
\bar{A}_{\varphi}^{*}(x, f)=\exp \left(\frac{1}{N} \sum_{n=1}^{N} \ln \frac{\left|Y_{\varphi}^{(n)}\right|}{\left|U_{\varphi}^{(n)}\right|}\right) .
$$

The amplitude spectra of the seismic motion $|S(f)|$ in direction $\varphi$ is evaluated from the complex spectra of the two horizontal components $S_{1}(f)$ and $S_{2}(f)$ as

$$
\left|S_{\varphi}\right|=\left|S_{1} \sin \varphi+S_{2} \cos \varphi\right| .
$$

In particular, we set $S_{I}=U_{I}^{(n)}$ and $S_{2}=U_{2}^{(n)}$ when evaluating $\left|U_{\varphi}^{(n)}\right|$ whereas $S_{I}=H_{11} U_{I}^{(n)}+H_{12} U_{2}^{(n)}+H_{13} U_{3}^{(n)}$ and $S_{2}=H_{21} U_{I}^{(n)}+H_{22} U_{2}^{(n)}+H_{23} U_{3}^{(n)}$ when evaluating $\left|Y_{\varphi}{ }^{(n)}\right|$. The polarization analysis consists in the computation of the directional amplification function for azimuth angles from $0^{\circ}$ to $175^{\circ}$ using a $5^{\circ}$ step.

\section{Rotation rate analysis from the $3 \times 3$ response function}

In consideration of the growing interest for the implications of the differential ground motion in seismic hazard (Guidotti et al. 2018), we attempted to analyze how topography and structure-topography interaction may induce torsional excitation (i.e., rotation around the vertical axis) at the Arquata del Tronto site. Given the ground velocity time series $\boldsymbol{v}(\boldsymbol{x}, \mathrm{t})=\left[v_{l}(\boldsymbol{x}, \mathrm{t}), v_{2}(\boldsymbol{x}, \mathrm{t}), v_{3}(\boldsymbol{x}, \mathrm{t})\right]$ in a grid of points $\boldsymbol{x}$ at surface, we follow the notation suggested in Evans and IWGoRS (2009) and describe the rotation around the vertical axis by means of the rotation rate

$$
\dot{\theta}_{3}(t)=\frac{1}{2}\left(\frac{d v_{2}}{d x_{1}}-\frac{d v_{1}}{d x_{2}}\right)
$$

In the case the ground motion is due to the passage of a plane wave, we can infer from equation (10) a proportionality between the rotational motion and the time derivative of the translational motion (Newmark 1969). If we denote with $\dot{\Theta}_{3}(f)$ the Fourier spectrum of the rotation rate around the vertical axis and with $\ddot{U}(f)$ the acceleration spectrum, we can write

$$
\left|\dot{\Theta}_{3}(f)\right|=\frac{s}{2}|\ddot{U}(f)|
$$

where $s$ denotes the horizontal slowness, i.e. the inverse of the apparent horizontal phase velocity of the propagating wave. The validity of equation (11) and of its equivalent in terms of peak ground rotation and peak horizontal velocity has been confirmed in field observations (e.g. Spudich and Fletcher 2008) and reproduced in numerical simulations (e.g. Stupazzini et al. 2009) in topography-free cases. Here we discuss instead the implications of equation (11) in the case of topographic features for a vertically emerging plane wave. A vertically emerging plane wave in a homogeneous medium with horizontal flat surface is characterized by $s=0$ and thus implies no rotation. On the other hand, heterogeneities and topographic features would imply local variations of $s$ and consequently may induce torsional motion as a site effect. Simple geometrical considerations allow us to deduce that in the ideal case of a homogeneous elastic medium with shear wave velocity $V_{s}$, the topographic surface with slope $\beta$ would imply for a vertically emerging plane wave an apparent horizontal slowness with value

$$
s=\frac{\tan \beta}{V_{s}} .
$$

If we substitute eq. (12) in eq. (11) we can predict the local (i.e., dependent on the position $\boldsymbol{x}$ ) scaling factor $A_{\theta}$ between the Fourier amplitude spectra of the rotation rate and the acceleration of the seismic input as

$$
A_{\theta}=\frac{\left|\dot{\Theta}_{3}\right|}{|\ddot{U}|}=\frac{\tan \beta}{2 V_{s}} .
$$

In the case of a layered medium, we can approximate $V_{\mathrm{S}}$ in eq. (13) with the weighted harmonic mean of $V_{\mathrm{S}}$ in the uppermost layers, but we have to expect a larger dispersion of the effective scaling factor in respect of the predicted one. 
In order to evaluate the effectiveness of equation (13) in the case of our 3D models, we apply eq. (10) to compute the rotation rate time series $\dot{\theta}_{3}^{(n)}(t)$ from the horizontal components $y_{1}^{(n)}(\boldsymbol{x}, t)$ and $y_{2}{ }^{(n)}(\boldsymbol{x}, t)$ of the ground velocity on the topographic surface, that are in turn estimated from the $n$-th seismic input using eq. (1). Finally, we evaluate the plane wave torsional scaling factor $\bar{A}_{\theta}^{*}$ as the geometric average of the ratio between the Fourier amplitude spectra of the rotation rate and of the horizontal acceleration of the seismic input

$$
\bar{A}_{\theta}^{*}(x, f)=\exp \left(\frac{1}{N} \sum_{n=1}^{N} \ln \left(\frac{\left|\dot{\Theta}_{3}^{(n)}(x, f)\right|}{\left|\ddot{U}^{(n)}(f)\right|}\right)\right)
$$

\section{The case study}

We used numerical modeling to estimate the site response in a dense grid of points distributed over a limited area centered on the location of the Arquata del Tronto village in Central Apennines (Italy).

\section{Geomorphological and lithological characters}

The geological substratum of the area is represented by the torbiditic member of the Laga formation consisting in an alternation of Arenaceous and Pelitic-Arenaceous lithofacies (Giallini et al. 2020; Amanti et al. 2020). The Laga formation is locally covered by fluvial deposits or arenaceous/calcareous debris (NE and SE area at the base of the Arquata crest) and is characterized by the alternation of stratified, and roughly $50^{\circ} \mathrm{WNW}$ dipping, different lithotypes belonging to the Laga formation. Instead, the substratum of Borgo in the valley below the hill presents 10 to 30 meters thick gravel deposits. In the description of the deep structure of the velocity model we consider two units (i.e. the pelitic and the arenaceous unit), whereas other two units are considered to describe the overall superficial $20 \mathrm{~m}$ thick alteration layer and the gravel deposits at Borgo. Further details regarding the set-up of the 3D geological model are given in Primofiore et al. (2020). We illustrate the morphological and lithological characteristics of the area with the four maps in Fig. 1. 

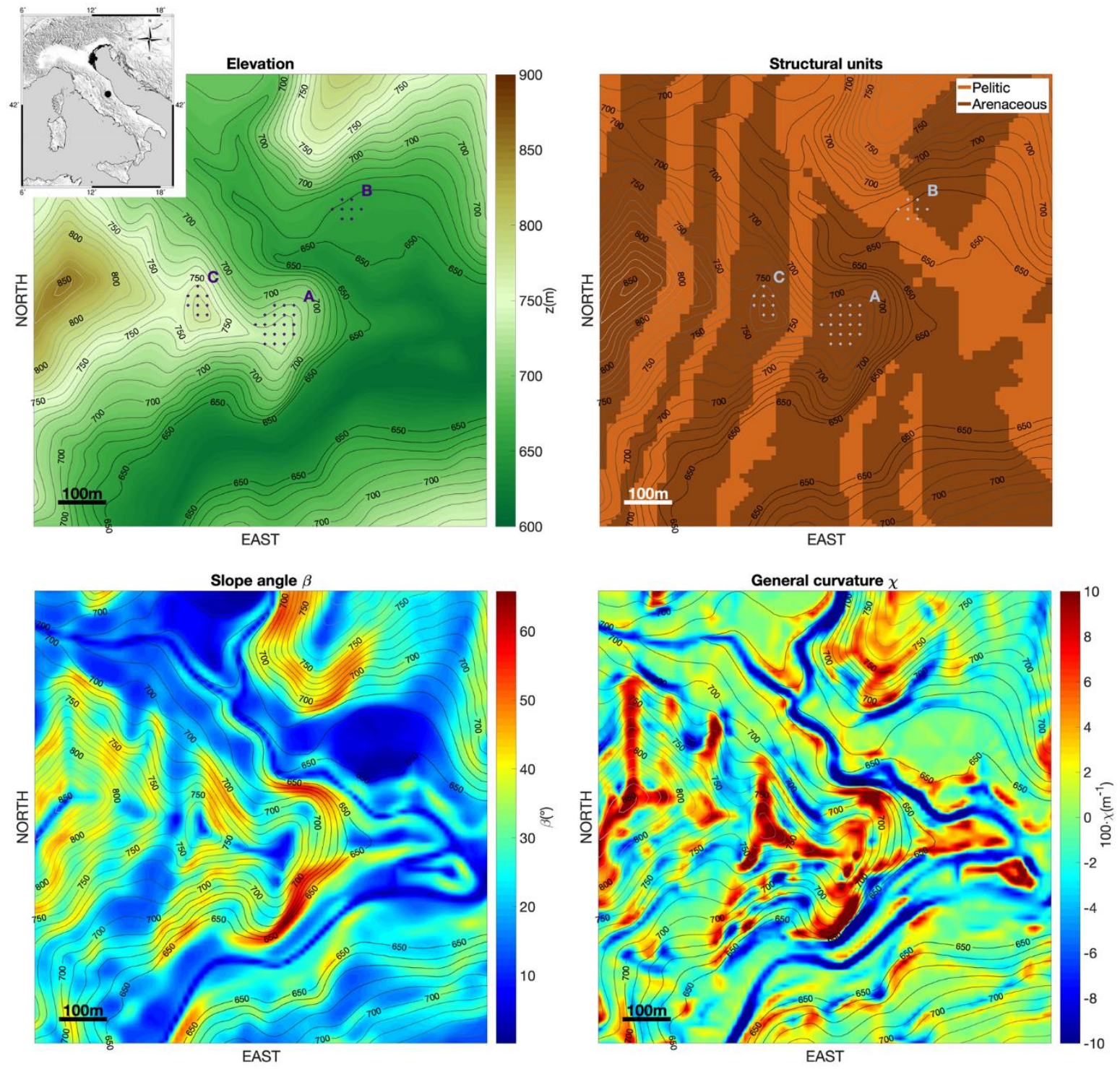

Fig.1 topographic and morphologic characterization of the studied area. The location of the area in the Italian peninsula is evidenced with the black dot in the small map overlapping the elevation map (upper left). The cross marks in the elevation map indicate the virtual seismic stations in three sites of interest: site A corresponds to Arquata del Tronto hamlet, site B to Borgo in the valley and Site C to the Castle promontory. In the structural model map (upper right) we depict the alternance of pelitic and arenaceous formations just below the weathering layer. The topographic slope and curvature are shown in the bottom left and right maps respectively

The elevation map evidences how the WNW-ESE oriented ridge of Arquata is transversely cut by saddles. The elevation stands in a range between 600 and $860 \mathrm{~m}$ a.s.l. In the structural units map we represent the distribution of the two deep structural units below the overall top alteration layer. In the same map we evidence the location of three sites of particular interest: Site A corresponds to the Arquata historical city center, Site B corresponds to the Borgo area in the valley and Site C corresponds to the Castle settlement on the crest. Site A and C are both placed on the Arquata ridge and lay above the same arenaceous formation, whereas site B, in the valley, mostly lays on the pelitic association with lower velocity (the gravel deposits that characterize site B are not shown in the map). The other two maps show the distribution of the topography's slope and general curvature. If we locally approximate the elevation $\mathrm{z}(\mathrm{x}, \mathrm{y})$ with the polynomial (Zevenbergen and Thorne 1987):

$$
z(x, y)=A x^{2} y^{2}+B x^{2} y+C x y^{2}+D x^{2}+E y^{2}+F x y+G x+H y+I
$$

The slope $\beta$ and the general curvature $\chi$ are defined (Moore et al. 1991) 


$$
\beta=\tan ^{-1} \sqrt{G^{2}+H^{2}} ; \quad \chi=-2(E+D)
$$

The general curvature can be used as a general measure of the land convexity, in the sense that the positive values indicate the convex surfaces (ridges and peaks), and the negative values identify concave surfaces, i.e. valleys.

The steepest slopes of the ridge are found on the East and North sides of the promontory with a slope angle of 60 degree, whereas we can identify the area of high convexity with the main WNW-ESE ridge and its NS oriented secondary ridges. Confronting the structural units map and the general curvature map, we observe that the NS oriented secondary ridges overlap the outcrop of the arenaceous formation.

\section{Numerical models}

We simulated the wave propagation in a spatial domain that represents a square area centered on the Arquata del Tronto hill, with $1 \mathrm{~km}$ long sides oriented along the geographical axes and that extends almost $1 \mathrm{~km}$ in depth. Computer-aided 3D geological modeling allowed us to partition the domain in four main structural units (Table 1, AdT2 model), consistently with the geological map and a number of geological vertical sections (ISPRA 2018).

Table 1 the structural units and their properties in the three geophysical models used for numerical simulations

\begin{tabular}{|l|l|c|l|l|l|l|l|}
\hline Model & Geological formation & Thickness $(\mathbf{m})$ & $\mathbf{V s}(\mathbf{m} / \mathbf{s})$ & $\mathbf{V} \mathbf{p}(\mathbf{m} / \mathbf{s})$ & $\mathbf{Q} \boldsymbol{\mu}$ & $\mathbf{Q \kappa}$ & $\boldsymbol{\rho}\left(\mathbf{k g} / \mathbf{m}^{\mathbf{3}}\right)$ \\
\hline \multirow{2}{*}{ AdT0 } & Bedrock & - & 1000 & 2480 & 100 & 350 & 2187 \\
\hline \multirow{2}{*}{ AdT1 } & Alluvial deposits & 25 & 450 & 1116 & 45 & 157 & 1792 \\
& Weathering layer & 25 & 700 & 1736 & 70 & 245 & 2001 \\
& Bedrock & - & 1000 & 2480 & 100 & 350 & 2187 \\
& & & & & & & \\
\hline \multirow{2}{*}{ AdT2 } & Alluvial deposits & 25 & 450 & 1116 & 45 & 157 & 1792 \\
& Weathering layer & 25 & 700 & 1736 & 70 & 245 & 2001 \\
& Pelitic association & - & 900 & 2232 & 90 & 315 & 2135 \\
& Arenaceous association & - & 1000 & 2480 & 100 & 350 & 2187 \\
& & & & & & & \\
\hline
\end{tabular}

In order to perform the numerical simulations we embedded the described 3D domain in a larger volume with 3 $\mathrm{km}$ long side square base aligned with the cardinal directions, extending from a depth of $500 \mathrm{~m}$ b.s.l. to the top surface which honors the $10 \mathrm{~m}$ resolution DEM Tinitaly (Tarquini et al. 2007). We discretized the spatial domain with an unstructured mesh, made of 518.400 hexahedral elements, using the SPECFEM3D internal mesher. We assigned to each element of the mesh the physical properties corresponding to the structural unit in its position. The elements' size is approximately $25 \mathrm{~m}$ near the top of the volume, where the low seismic velocities that characterize the alteration layer and alluvial deposits imply shorter wavelengths. On the other hand, we set an element size of $50 \mathrm{~m}$ in the lower part of the volume, where seismic velocities are higher. A $75 \mathrm{~m}$ thick Perfectly Matched Layer was applied on the lateral and bottom boundaries of the volume in order to exclude artefact reflections from the numerical solution. The numerical mesh, clipped on the central $1 \mathrm{~km}^{2}$ study area is presented in Figure 2.

To investigate the separate contribution of stratigraphic and topographic effects on the seismic ground motion, we carried out our analysis on three different geophysical models (Table 1 and Figure 3): AdT0, AdT1 and AdT2. AdT0 presents a homogeneous structure and is therefore characterized by topographic effects alone. AdT1 includes a $25 \mathrm{~m}$ thick weathering layer below the surface and the alluvial deposits in the small basin at site B whereas AdT2 includes also the distinction between the arenaceous and pelitic associations in the deeper structure. 


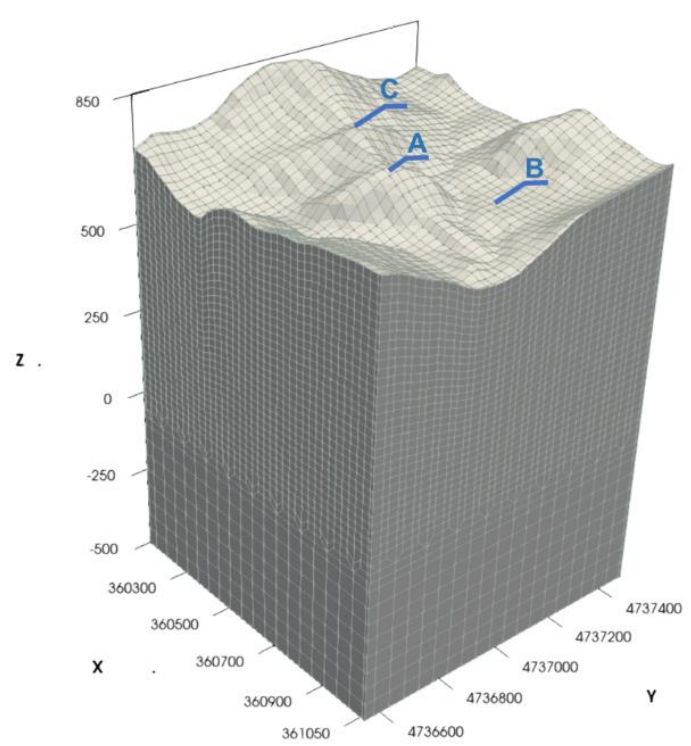

Fig.2 the central portion of the numerical mesh used for numerical simulations. The locations of the sites $A, B$ and $C$ are shown. Length units are in meters, $x$-direction is eastward

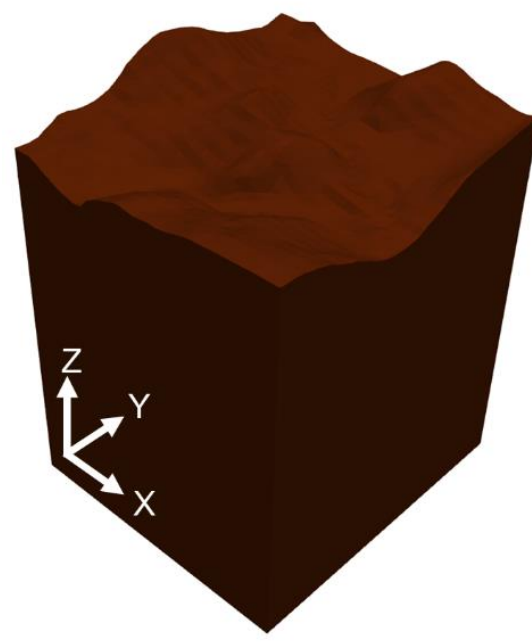

AdTO

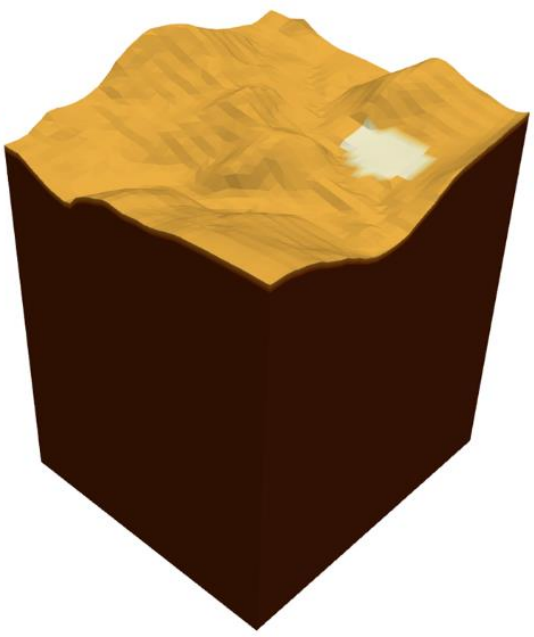

AdT1

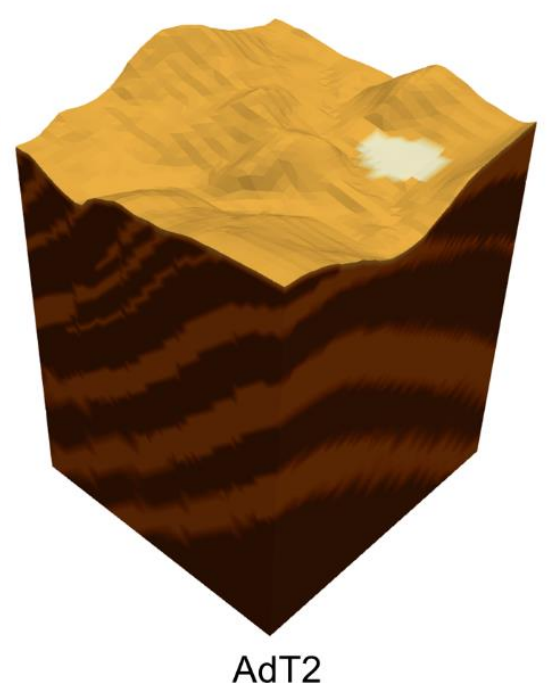

AdT2

Fig.3 representation of the three input models clipped on the $1 \mathrm{~km}^{2}$ square area of interest. AdTO is the homogeneous model, AdT1 is the homogeneous model with a weathering layer and an alluvial, AdT2 is the full $3 D$ model of Arquata del Tronto retrieved from Primofiore et al. (2020)

We modelled the vertically incident plane waves by placing a horizontal carpet of sources at $-400 \mathrm{~m}$ a.s.1, with an explosive mechanism for the vertically polarized plane wave and West-East or North-South oriented double couple mechanism for the two independent cases of horizontally polarized plane waves, respectively. A set of three simulations is therefore necessary to fully define the $3 \mathrm{D}$ response function. We performed simulations that returned $8 \mathrm{~s}$ long three-component time series at 2304 points of the computational mesh in the central square area of the topographic surface.

Considering the values of the mechanical properties (Table 1), the dimensions of the mesh elements imply a time step as short as $0.0005 \mathrm{~s}$ to satisfy the stability criterion of the time marching step implemented in the SPECFEM3D solver and accurate solutions of seismic waves up to the frequency of $10 \mathrm{~Hz}$ can be obtained. On the other hand, the finiteness of the overall size of the considered spatial domain undermines the meaningfulness of the modelling results for frequencies below $1 \mathrm{~Hz}$. Each simulation consisted therefore in 16000 time iterations with a computational cost amounting to approx. 900 CPU hours on 225 cores on the Galileo supercomputer at CINECA. 
388 In order to identify the possible site effects that are due to surface morphology alone, we first computed the numerical response functions (see eq. 3) for a homogeneous medium (model AdT0 in Table 1 and in Figure 3) and evaluated over the topographic surface the geometric averages of the site response parameters defined in the Method section. Successive computations regarding inhomogeneous media (models AdT1 and AdT2 in Table 1 and in Figure 3) allowed us to estimate the possible aggravations induced by the interaction between topography and stratigraphy. In order to evaluate the meaningfulness of the aggravations in the spectral amplification due to the changes in the model AdT $x$ in respect to the reference model AdT0, (where AdT $x$ stays for AdT1 or AdT2), we estimated the effect size, defined as

$$
\Delta^{(\mathrm{AdTx})}=\frac{\bar{A}_{l n}^{(\mathrm{AdTx})}-\bar{A}_{l n}^{(\mathrm{AdT} 0)}}{\sigma_{l n}^{(\mathrm{AdT} 0)}},
$$

with $\bar{A}_{l n}$ and $\sigma_{l n}$ defined in equation (6). In consideration of the frequency dependence of the site response, we summarize the results with maps representing the average value of the site response parameters functions over defined frequency bands. In particular we considered the averages over the following frequency (period) bands: $1-2 \mathrm{~Hz}(0.5-1 \mathrm{~s}), 2-4 \mathrm{~Hz}(0.25-0.5 \mathrm{~s})$ and $4-8 \mathrm{~Hz}(0.125-0.25 \mathrm{~s})$.

\section{Amplification}

In the homogenous medium hypothesis (model AdT0), the topographic features at Arquata del Tronto generate amplification effects in all three considered frequency ranges, as can be observed from the maps exposed in Figure 4. As expected, the amplification at a given frequency is controlled by topographic features with characteristic sizes comparable with the associated wavelength (Geli et al. 1988). The average amplification computed in the 1-2 Hz band correlates well with larger topographic features such as the whole Arquata hill, while amplification in the $4-8 \mathrm{~Hz}$ band correlates well with smaller topographic features such as the top ridge of Arquata and its N-S oriented secondary crests. 

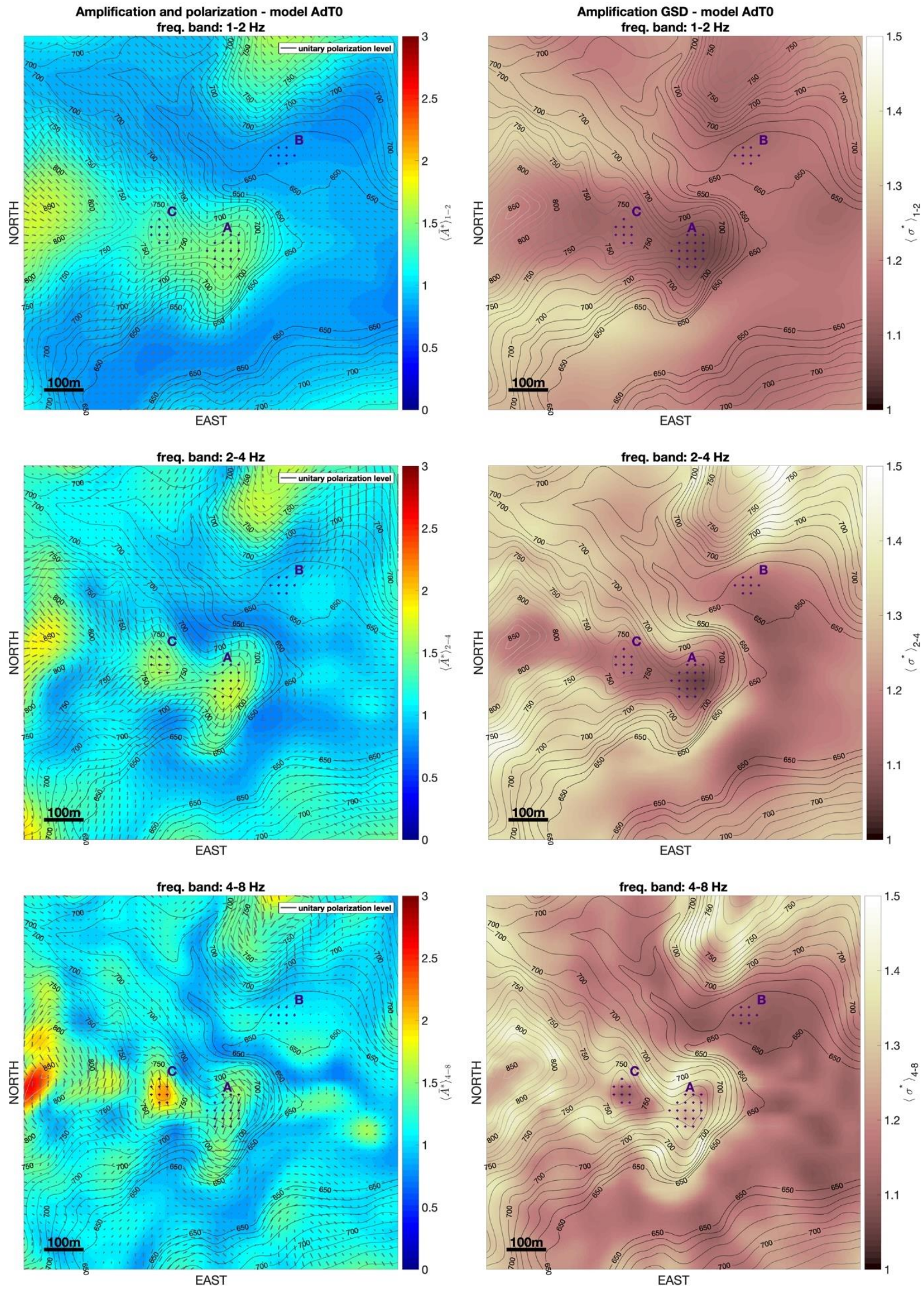

Fig.4 left) distribution of horizontal plane-wave amplification and polarization computed for the homogeneous model in the three frequency bands $1-2 \mathrm{~Hz}, 2-4 \mathrm{~Hz}$ and $4-8 \mathrm{~Hz}$. Grey headless arrows in the maps in the left 
Because of flat topography site B is unaffected by amplification in the considered bands, whereas sites A and C appear most affected in the $2-4 \mathrm{~Hz}$ and $4-8 \mathrm{~Hz}$ bands, respectively. The amplification however just exceeds the value 2 in the latter case. The geometric standard deviation is relatively low in the valley and along the top of the ridge in a strip whose width progressively decreases with frequency and almost vanishes in the highfrequency band.

The qualitative correspondence between topographic features and the response function amplitudes suggests that typical topographic effects could be speedily estimated from local morphometric variables. A promising approach in this sense is the Frequency-Scaled Curvature (FSC) introduced by Maufroy et al. (2015), who found a correlation between the seismic ground-motion amplification estimated with the median reference method MRM (Maufroy et al. 2012) and the general curvature $\chi$ (defined in equation 16), with a wavelength-dependent smoothing applied on it. In analogy to Maufroy et al. (2015) we compared the plane wave amplification with the smoothed general curvature for different values of the smoothing length. We used the SAGA algorithm (Conrad et al. 2015) to evaluate the general curvature $\chi$ at the surface points of the spatial domain and applied a $n \times n$ smoothing kernel, where $n$ is an odd number which defines the smoothing length as $L_{s}=2 n$ Dx where Dx is the resolution of the DEM (10 $\mathrm{m}$ in our case).

Our comparison consists in the evaluation of the linear correlation coefficient $r$ between the natural logarithm of the average value of the amplification function in the three frequency bands and the smoothed general curvature $\chi_{(\mathrm{Ls})}$, where we express the smoothing length $L_{s}$ in terms of the central wavelength $\lambda_{\mathrm{c}}$, defined as the geometric mean of the wavelengths corresponding to the band extremes. From Fig. 5 it appears that the maximum correlation corresponds to a smoothing length $L_{s}$ between $0.5 \lambda_{\mathrm{c}}$ and $0.8 \lambda_{\mathrm{c}}$. This result is consistent with Maufroy et al. (2015), who established a maximum correlation between MRM amplification and smoothed curvature for a smoothing length of about half the wavelength. In the lower part of Fig. 5 we provide the weighted least squares linear regression between $\left\langle\bar{A}_{l n}\right\rangle_{f 1-f 2}$ and $\chi_{(\mathrm{Ls})}$ with $L_{s}$ corresponding to the maximum correlation case. The coefficient of determination $R^{2}$ reaches 0.8 in the $1-2 \mathrm{~Hz}$ frequency band but at higher frequencies the dispersion increases and $R^{2}$ drops to 0.57 and 0.54 for the $2-4 \mathrm{~Hz}$ and $4-8 \mathrm{~Hz}$ band respectively. In Fig. 6 we can see how the horizontal amplification functions in the three sites A, B and C change because of the introduction of complexities in the model structure. The most remarkable changes concern site B, which is characterized by flat topography. The introduction of soil basin increases the amplification at site B to higher values than the topography-related amplification at sites $\mathrm{A}$ and $\mathrm{C}$.

In the maps on the left side of Fig. 7 we represent the spatial distribution of $\left\langle\bar{A}^{*}\right\rangle_{f_{1}-f_{2}}$ for the model AdT1, and on the right side the corresponding effect size in respect to the model AdT0.

The AdT1 model differs from AdT0 in $25 \mathrm{~m}$ thick weathering layer just below the surface over all the area and a 10 to $30 \mathrm{~m}$ thick layer of gravel deposits in the alluvial basin in the restricted area of Borgo (site B).

The weathering layer leads to a general increase of the amplification, with modulations that follow the topographic features. In the low frequency band $(f<2 \mathrm{~Hz})$ the increase in the amplification is not significant at any point, whereas in the intermediate and high frequency bands the amplification gain significance at some spots, but not on the flanks of the hills. The intermediate and high frequency amplification associated with the introduction of the soil layers in the alluvial basin at site B exceeds any amplification related to the interaction between topography and the weathering layer. The absence of amplification aggravation in the low frequency band can be easily explained if we consider that we assigned $\mathrm{Vs}=450 \mathrm{~m} / \mathrm{s}$ to the soil in the basin with a maximum depth of $30 \mathrm{~m}$, which implies a stratigraphic amplification effects for frequencies above $3.75 \mathrm{~Hz}$, whereas if we consider the combined effect of the basin and the weathering layer, the stratigraphic amplification does not affect frequencies below $2.5 \mathrm{~Hz}$.

In Fig. 8 we represent an analogous comparison between the results concerning model AdT2 and model AdT0. The increase in the amplification that is due to the introduction of the deep structure (model AdT2) presents a pattern that is quite similar to the model AdT1, but with an overall effect size which is only slightly larger than in the AdT1 case.

In order to emphasize the differences in the effects of the models AdT2 and AdT1 compared to the model AdT0, we plot in Fig. 9 a histogram of the effect size values in the points on the map in the three considered frequency bands. The models AdT2 and AdT1 present differences in the amplification in the lowest frequency bands (1-2 $\mathrm{Hz}$ and 2-4 Hz), whereas in the high frequency band $(4-8 \mathrm{~Hz})$ the two models are essentially equivalent. The broader distribution of the effect size values for the high frequency band in Fig. 9, can be explained with the sensitivity of the seismic response at high frequencies to small scale topographical and geological features. 

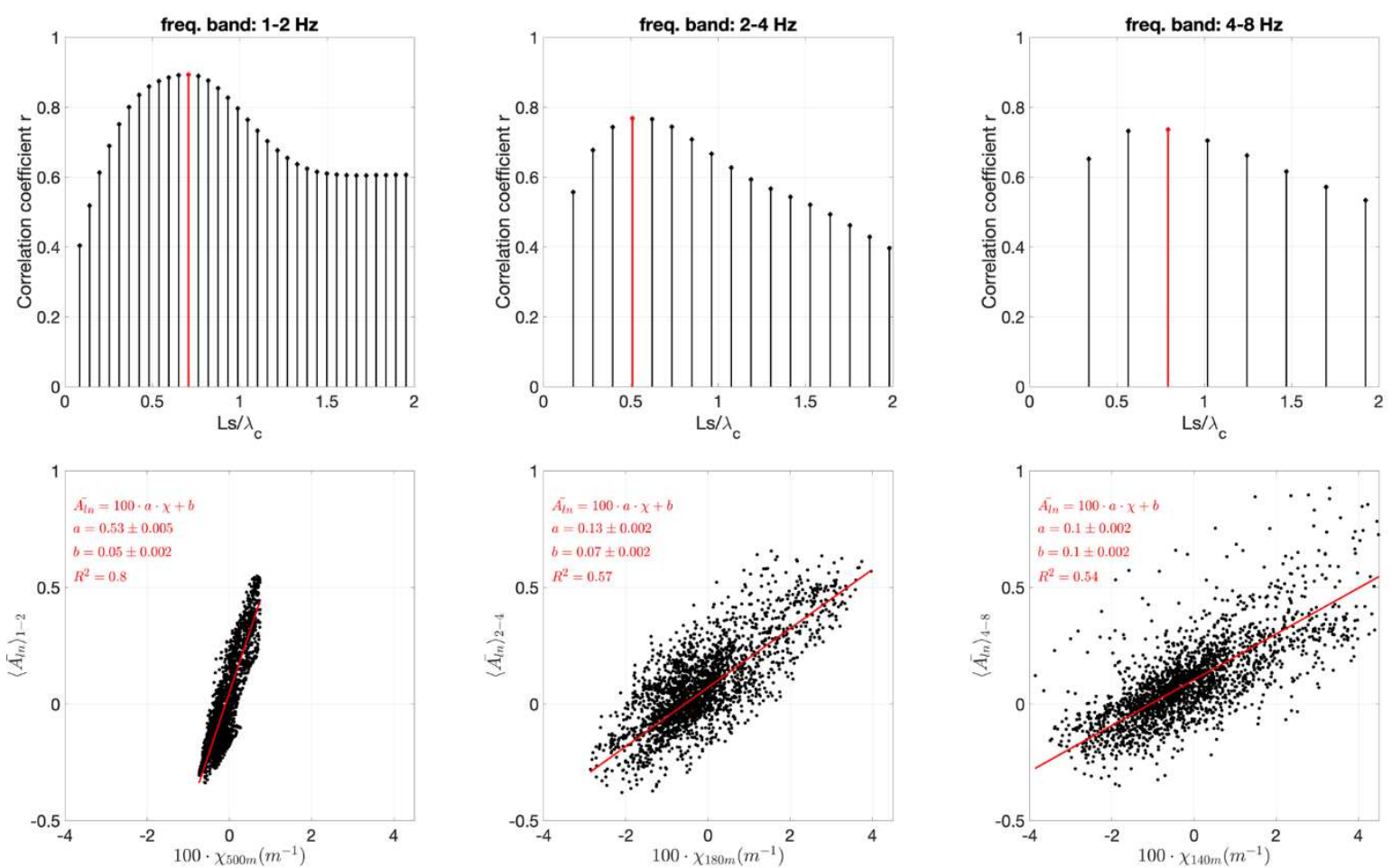

Fig.5 top) correlation coefficients between logarithmic plane-wave amplification $\left\langle\bar{A}_{l n}\right\rangle_{f 1-f 2}$ and smoothed curvature $\chi$ in three frequency bands for different values of the smoothing length Ls; bottom) weighted-least square regression analysis between $\left\langle\bar{A}_{l n}\right\rangle_{f 1-f 2}$ ) and smoothed $\chi$ with smoothing length Ls corresponding to maximum correlation for each frequency band
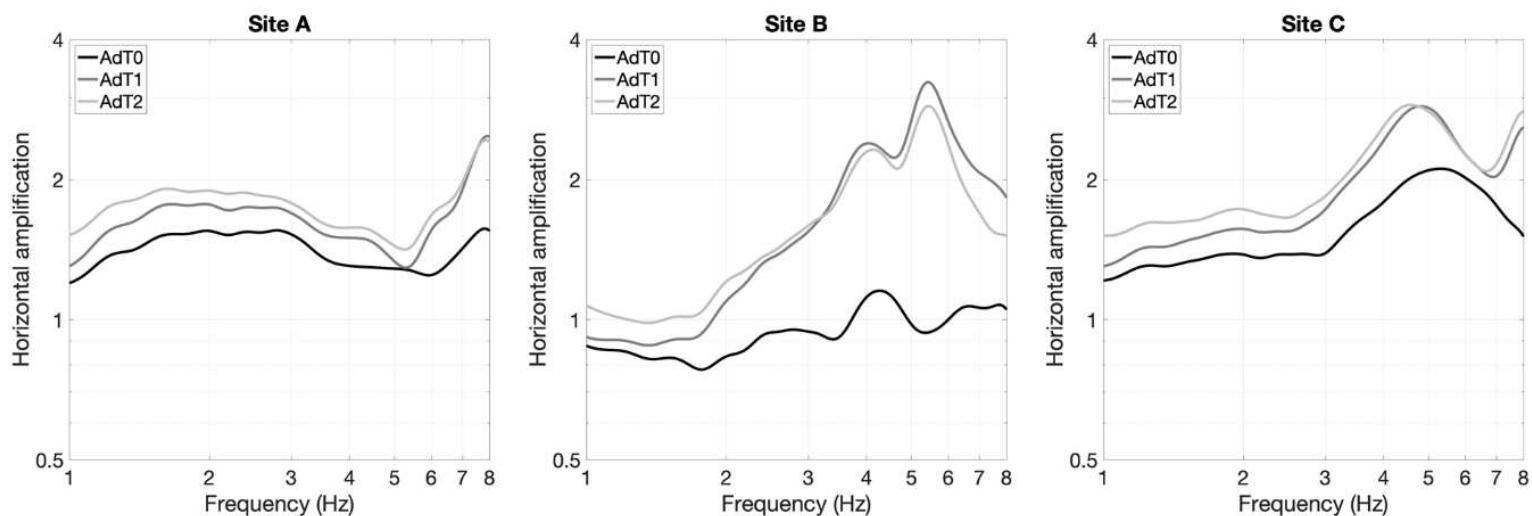

Fig.6 average horizontal plane-wave amplification at Site A (Arquata center), Site B (Borgo) and Site C (Arquata castle) for homogeneous AdTO model (black), inhomogeneous AdT1 model (dark grey) and 3D model AdT2 (light grey). The average implies the stations covering each site, indicated as crossmarks in the maps in Fig. 4 

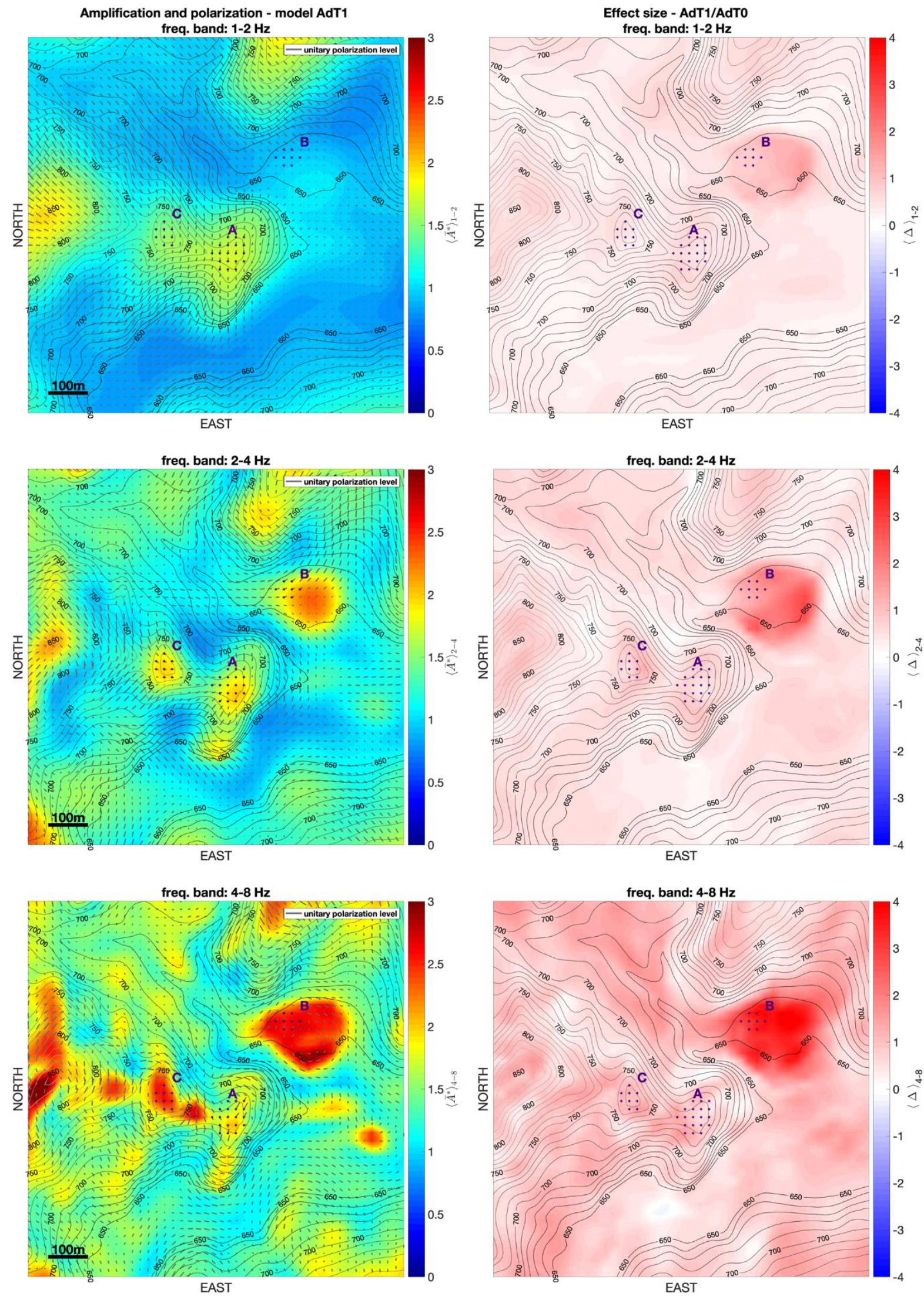

Fig.7 left) horizontal plane-wave amplification distribution pattern at Arquata del Tronto computed for the model AdTl and in the frequency bands 1-2Hz, 2-4Hz and 4-8Hz. Same notation as in Fig.4; right) the effect size (see Eq. 17 in the text) in the amplification values for the model AdT1 in respect to model AdTO 

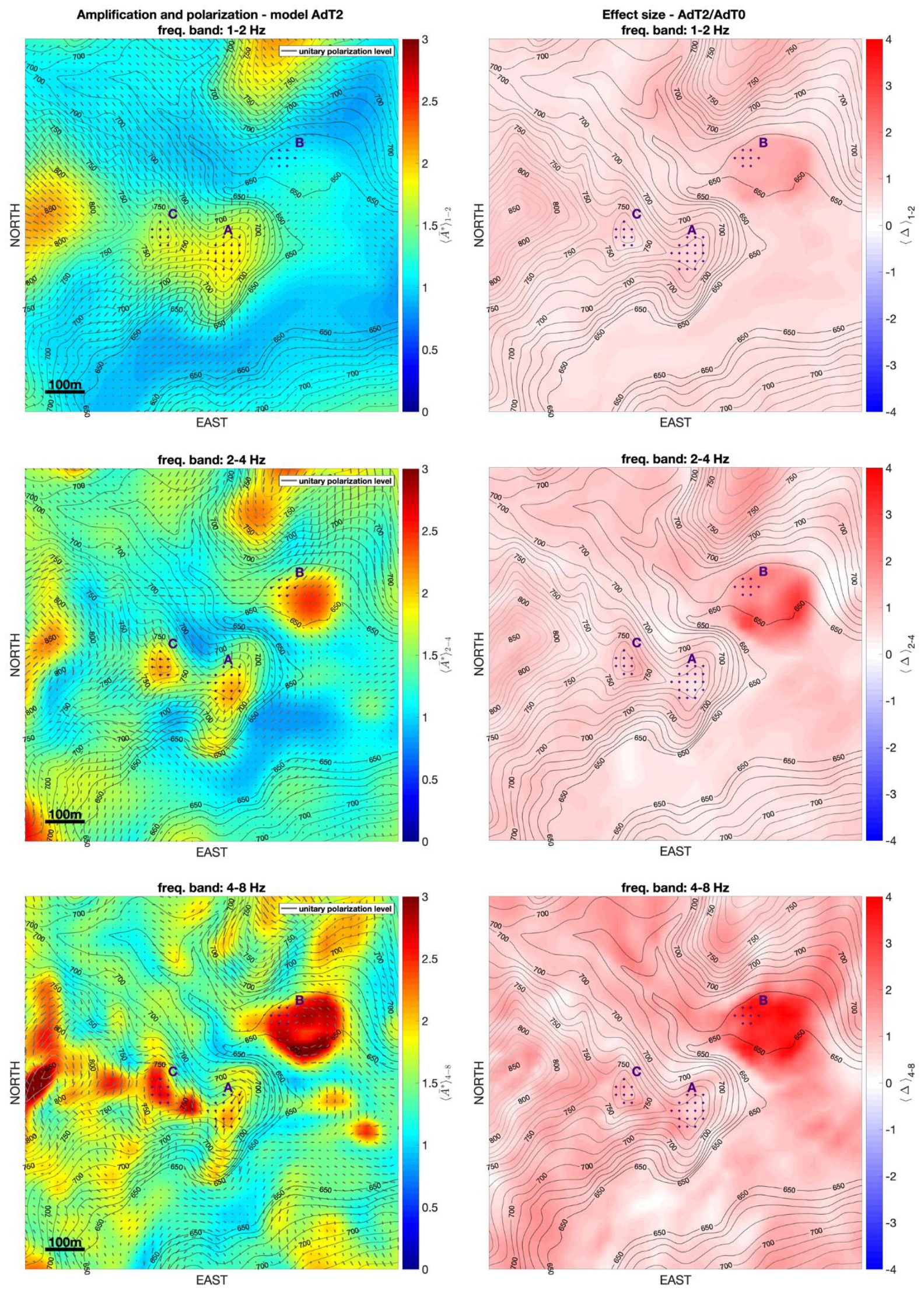

Fig.8 left) horizontal plane-wave amplification distribution pattern at Arquata del Tronto computed for the model AdT2 and in the frequency bands 1-2Hz, 2-4Hz and 4-8Hz. Same notation as in Fig.4; right) the effect size (see Eq. 17 in the text) in the amplification values for the model AdT2 in respect to model AdTO 

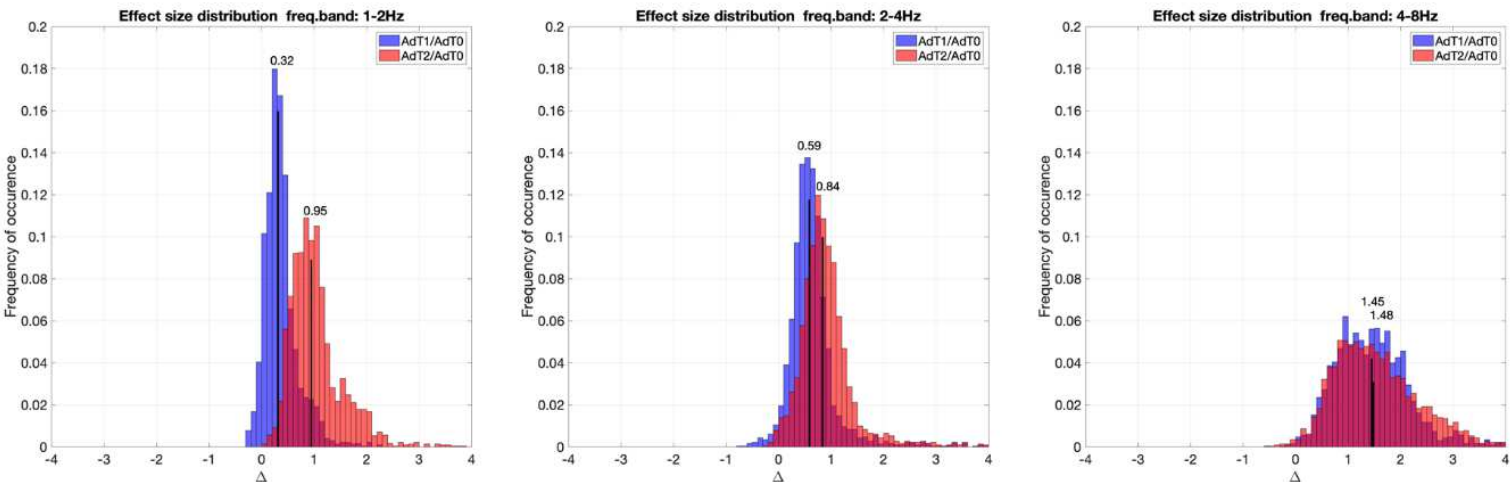

Fig.9 histograms of the amplification effect size values over the grid points sampling the studied area for the frequency bands: $1-2 \mathrm{~Hz}, 2-4 \mathrm{~Hz}$ and $4-8 \mathrm{~Hz}$. The median value is indicated in order to assess the overall differences between the changes in the amplification functions due to the variations in the structural models

With the introduction of inhomogeneity in the model we found a frequency dependent drop in the correlation between the smoothed curvature and average amplification. In Figure 10 we plot the highest correlation scores for all the three investigated models in the three frequency bands. We note that in the $1-2 \mathrm{~Hz}$ band the heterogeneity in the model has almost no effect on the correlation between the amplification and the smoothed curvature. On the other hand, the variations in the subsurface model reduce substantially the correlation in the other two frequency bands, with the $4-8 \mathrm{~Hz}$ band being the most sensible to the subsurface structure effects, but without significant distinction between the two heterogeneous models.

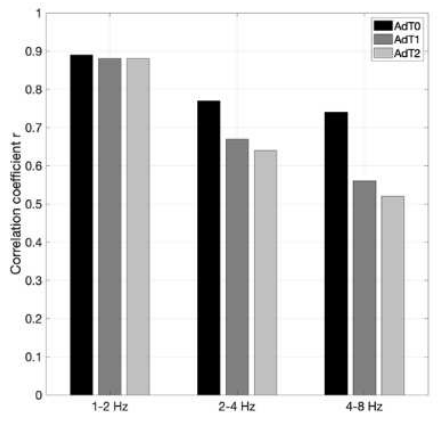

Fig.10 correlation coefficients between averaged horizontal plane-wave amplification and smoothed curvature obtained in the three frequency bands of interest for homogeneous model AdTO (black), inhomogeneous model AdT1 (dark grey) and 3D model AdT2 (light grey)

\section{Horizontal Polarization}

The distribution of the horizontal polarization in the site response for the model AdT0 is summarized on the maps on the left side in Fig.4, with the headless arrows indicating the direction $\varphi_{\max }$ of the maximum directional amplification averaged over the three frequency bands and the arrows' length indicating the polarization level defined as

$$
P=1-\frac{A_{\varphi_{\min }}}{A_{\varphi_{\max }}},
$$

with $A_{\varphi_{\min }}$ and $A_{\varphi_{\max }}$ the minimum and the maximum directional amplifications at each point. $P$ is zero when the amplification is isotropic and tends to 1 for increasing variability in the directional amplification. We can observe that the direction and level of polarization change significantly in the three considered frequency bands. As can be seen from Fig.11 and Fig.12, the amplification appears to be generally uncorrelated with the polarization level: in many points the polarization of the response corresponds to directional deamplification rather than amplification. This is the case of the polarization level found along the northern and 
the southern slope of the Mt. Arquata ridge in the 1-2 Hz and 2-4 Hz bands. In the 2-4 Hz band the polarization level appears to be larger in the portion of the ridge's flanks that is closer to the top, but actually no clear relation between the polarization direction and the topographic features can be observed at these points, where polarization does not imply amplification. On the other hand, the points that are characterized by both polarization and amplification are essentially located on the primary and (in the 4-8 Hz band) secondary ridges. In this case the polarization direction is typically perpendicular to the crest orientation. We can therefore conclude that even if the topography-induced amplification is associated with polarization, the polarization itself does not necessarily implies topography-induced amplification.

The introduction of the weathering layer and deeper structural units (Fig.7 and Fig.9, respectively), does not bring noticeable changes in the polarization pattern in the $1-2 \mathrm{~Hz}$ and 2-4 Hz bands, whereas in the 4-8 $\mathrm{Hz}$ band we can observe a reduction of the polarization level at site $\mathrm{A}$ on the edge of the ridge and the appearance of polarization in the seismic response at the edges of the nearly circular soil basin near site B, with direction tangential to the basin edge. The heterogeneous structure may therefore attenuate the topography-related polarization effects (without reducing the amplification) on one side and can produce topography-unrelated amplification and polarization, on the other.
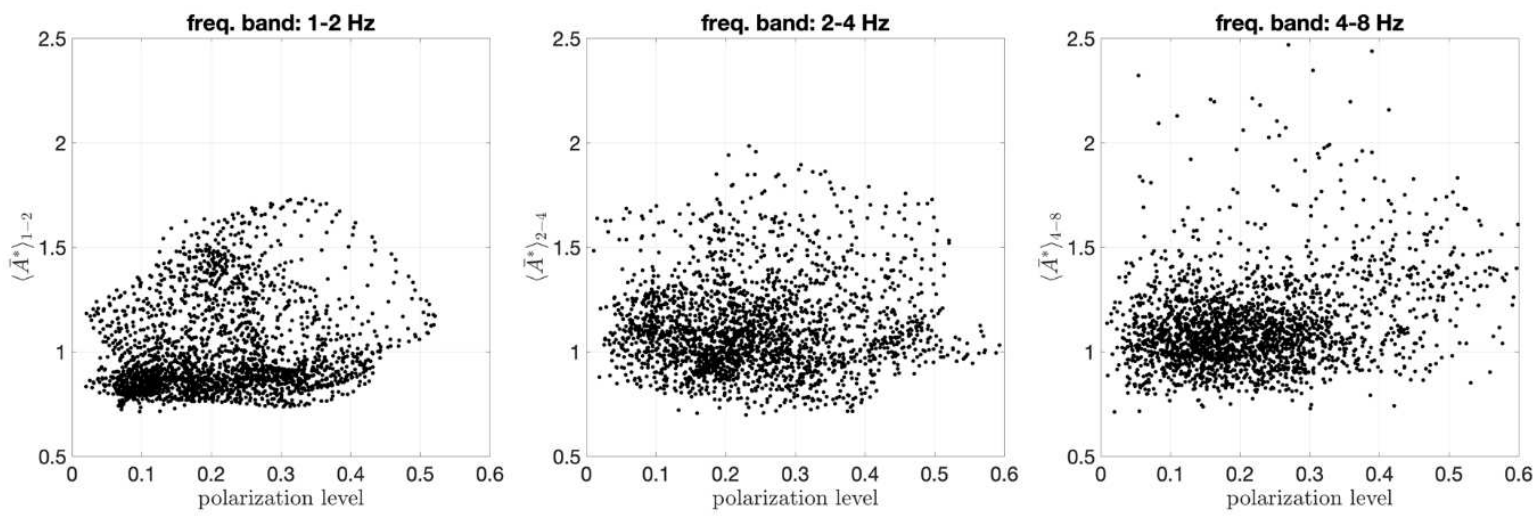

Fig.11 distribution of amplification values in function of the polarization level in the three frequency bands for the Model AdTO case

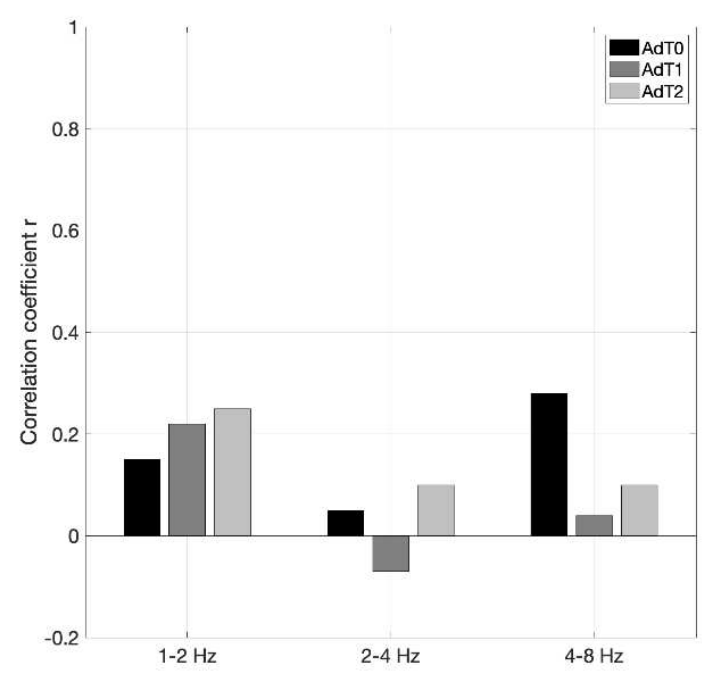

Fig.12 the values of the linear correlation coefficient between the polarization level and amplification

\section{Torsional motions}

In Fig. 13 we plot, for the three models, the maps corresponding to the average over the 4-8 $\mathrm{Hz}$ frequency band of the parameter $\bar{A}_{\theta}^{*}$ defined in equation (14), which describes the scaling factor between the site-induced 
angular velocity around the horizontal axis (i.e. torsional motion) and the translational acceleration of the vertically incident plane wave. From the map corresponding to the homogeneous medium (model AdT0) it appears that the highest values of the induced rotational motion are associated with the points on topographic slopes, as predicted by the conceptual model that lead us to equation (13). The variability in $\left\langle\bar{A}_{\theta}^{*}\right\rangle_{4-8 H z}$ is described by a multiplicative standard deviation which ranges between a factor 1.5 and 2 .

Maps regarding the frequency ranges $1-2 \mathrm{~Hz}$ and 2-4 $\mathrm{Hz}$ are available in the supplemental information as Figs. $\mathrm{S} 3$ and S4.

From the maps regarding model AdT1 and model AdT2 in the frequency range 4-8 Hz (Fig.13), it is clear that the introduction of the weathering layer enhances the rotational motion on the slopes whereas the introduction of the basin structure produces rotations near the basin edges. These structure-induced rotations are of the same order of magnitude of those induced by the concurrence of topography and weathering. We can also notice that since they are characterized by low slope, the sites $\mathrm{A}$ and $\mathrm{C}$ are not affected by the appearance of torsional components in the ground motion.
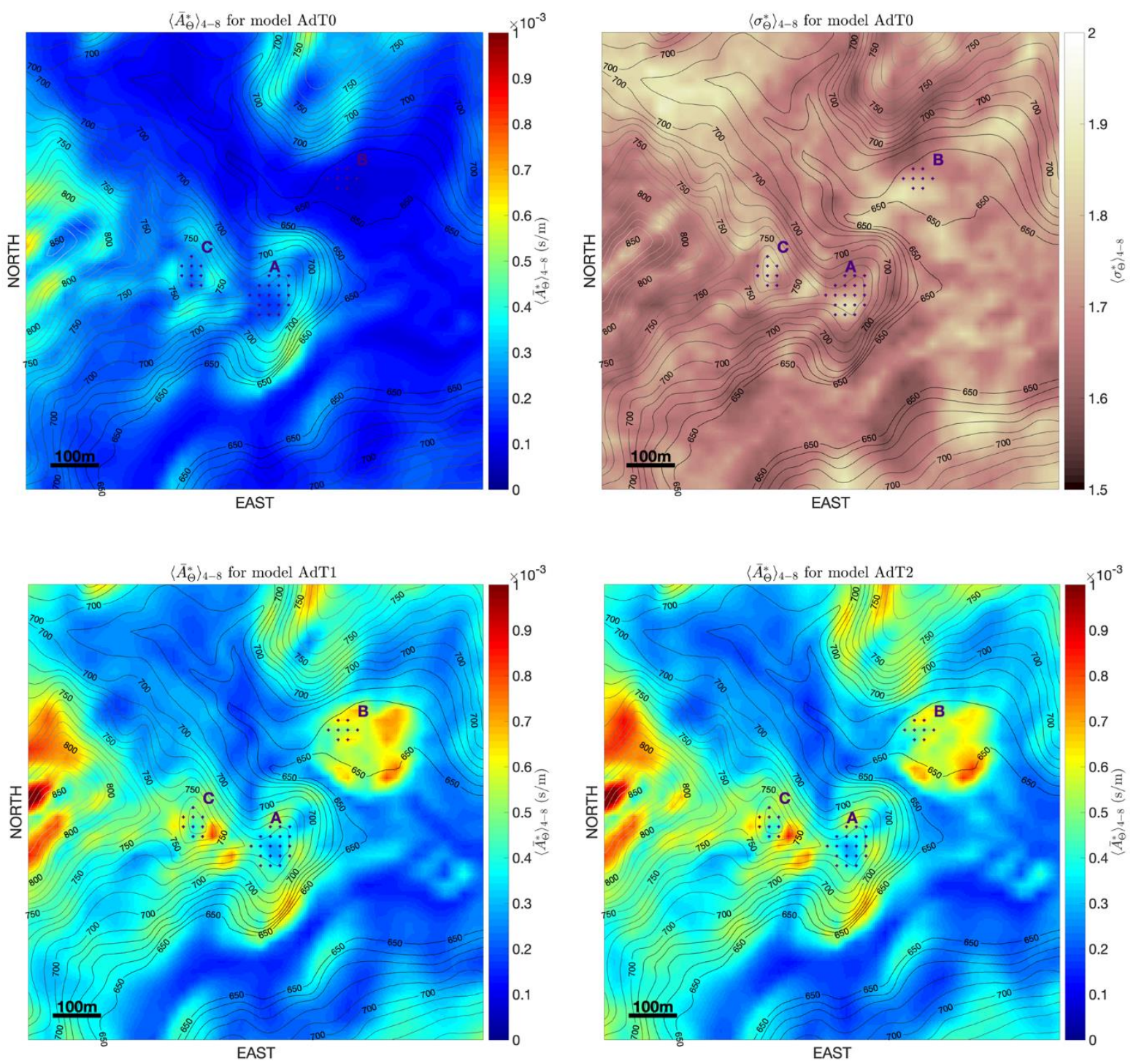

Fig.13 scaling factor $\bar{A}_{\theta}^{*}$ between the site-induced torsional motion and the translational acceleration of the vertically incident plane wave, averaged in the frequency band 4-8Hz for the three models AdTO, AdT1 and $A d T 2$ and respective GSD $\sigma_{\theta}^{*}$ for model AdTO

To verify quantitatively if equation (13) could be used to estimate the rotational scaling factor $\left\langle\bar{A}_{\theta}^{*}\right\rangle_{4-8 \mathrm{~Hz}}$ computed from the three models, we apply the weighted least squares approach to find the parameters a and $\mathrm{b}$ in the linear fit 


$$
\left\langle\ln \bar{A}_{\theta}^{*}\right\rangle_{4-8 H z}=a \ln [\tan \beta]+b .
$$

We consider the slope $\beta$ distribution evaluated from the topography with the SAGA algorithm (Conrad et al. 2015), and apply a smoothing with length $125 \mathrm{~m}$, corresponding to the wavelength of the incident waves at the maximum considered frequency ( $8 \mathrm{~Hz}$ ). We neglect the points with slope $\beta<15^{\circ}$ in order to exclude from the analysis the rotation induced in the basin area near site B.
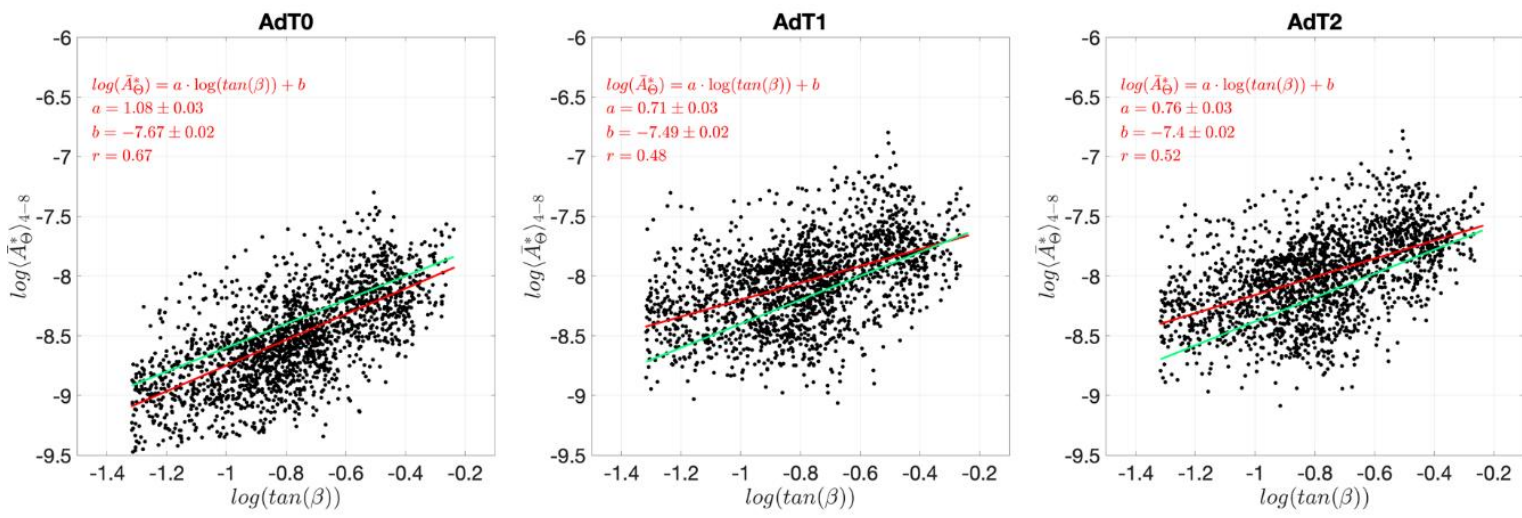

Fig.14 weighted-least square regression (red line) between $\left\langle\ln \bar{A}_{\theta}^{*}\right\rangle_{4-8 \mathrm{~Hz}}$ and $\ln [\tan (\beta)]$ at smoothing length $L s=125 \mathrm{~m}$, for the three models in the high frequency band $(4-8 \mathrm{~Hz})$. The green line indicates the expected regression (parameters $a$ and $b$ indicated in parenthesis in Tab.2)

The linear fit is shown in Fig. 14 and the parameters $a$ and $b$ corresponding to the three models are tabulated in Table 2 along with the $\mathrm{V}_{\mathrm{S}}$ value interpreted from the $b$ parameter. In parenthesis we give the target values consistent with equation (13).

Table 2 results of the weighted-least square analysis between $\bar{A}_{\theta \text { ln }}^{*}$ and $\log (\tan (\beta))$ for the three models. Expected values for parameters $a$ and $b$ are given in parenthesis

\begin{tabular}{|l|l|l|l|}
\hline & AdT0 & AdT1 & AdT2 \\
\hline$a$ & $1.08(1.00)$ & $0.71(1.00)$ & $0.76(1.00)$ \\
\hline$b$ & $-7.67(-7.6)$ & $-7.49(-7.40)$ & $-7.40(-7.38)$ \\
\hline $\mathrm{Vs}=0.5 \exp (-\mathrm{b})$ & $1071 \mathrm{~m} / \mathrm{s}(1000.0 \mathrm{~m} / \mathrm{s})$ & $895 \mathrm{~m} / \mathrm{s}(825 \mathrm{~m} / \mathrm{s})$ & $818 \mathrm{~m} / \mathrm{s}(806 \mathrm{~m} / \mathrm{s})$ \\
\hline
\end{tabular}

Regardless the relatively high dispersion of the $\left\langle\ln \bar{A}_{\theta}^{*}\right\rangle_{4-8 H z}$ values (linear correlation $\mathrm{r}=0.67$ ), the AdT0 model provides a fit which is quite consistent with equation (13). As expected, the AdT1 and AdT2 models provides $\left\langle\ln \bar{A}_{\theta}^{*}\right\rangle_{4-8 H z}$ values that are less correlated with the topographic slope. Even that the values of the parameter $b$ for these two models are not too far from the ones corresponding to the harmonic mean of the Vs values in the weathering layer and in the underlying rock, the values of parameter $a$ significantly deviate from unity, implying higher values of the scaling factor $\bar{A}_{\theta}^{*}$ at moderate slope angles. In the cases where the topography effect combines with stratigraphic effects, we would need a more sophisticated predictive model than that provided in equation (13).

In figure 15 we analyze the differences between the effect size corresponding to the substitution of the AdT0 model with models AdT1 and AdT2, respectively. Only in the 4-8 Hz frequency band the median value of the effect size approaches unity, but in this frequency band the differences in the effect size of the two models are minimal. As in the amplification case, the $4-8 \mathrm{~Hz}$ band results the most sensible to the subsurface structure effects, without significant distinction between the two heterogeneous models. Even though the two heterogeneous models brings different levels of size effects in the other two frequency bands, these levels are below unity and could be considered less significant. The homogeneous model assumption is therefore sufficient to evaluate induced rotations in the frequency band up to $4 \mathrm{~Hz}$. 

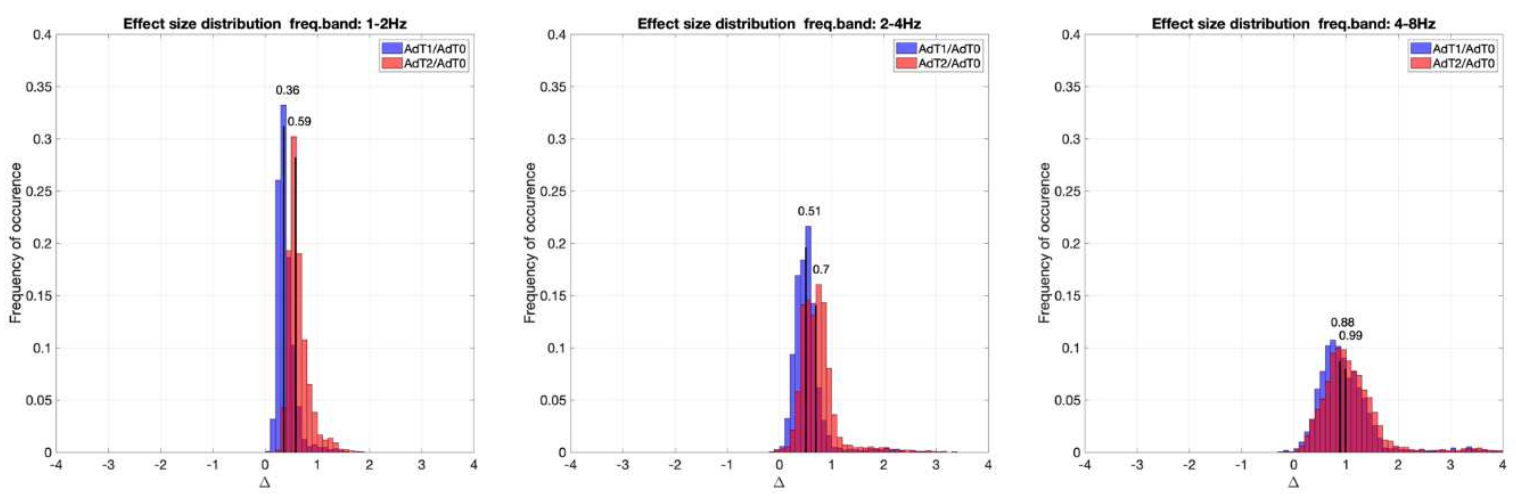

Fig.15 histograms of the induced torsional motion $\bar{A}_{\theta}^{*}$ effect size values over the grid points sampling the studied area for the frequency bands: $1-2 \mathrm{~Hz}, 2-4 \mathrm{~Hz}$ and $4-8 \mathrm{~Hz}$. The median value is indicated in order to assess the overall differences between the changes in the amplification functions due to the variations in the structural models

\section{Conclusions}

In order to shed light on the three dimensional seismic local effects suffered by the Arquata del Tronto ridge under the 2016 Central Italy seismic sequence we set up a 3D numerical model of the area and computed the linear visco-elastic site response for a vertically incident plane wave by using the well-established software SPECFEM3D. In particular we analyzed the separated and combined effects of irregular topography and subsurface heterogeneity on the seismic ground motion in terms of horizontal component spectral amplification, including the aspects that concern polarization and rotation. To facilitate the discussion, we averaged the results over the frequency (period) bands of engineering interest: 1-2 Hz (0.5 -1 s or low frequency), 2-4 Hz (0.25-0.5 s or intermediate frequency) and $4-8 \mathrm{~Hz}(0.125-0.25 \mathrm{~s}$ or high frequency). By comparing the numerical results based on three different geophysical models of the area, one homogeneous, one with a weathering layer and one with a complex 3D structure, we were able to distinguish typical topographic effects from combined effects of topography and stratigraphy. We evaluated the intrinsic variability in the 3D seismic response caused by crosscoupling of different components of the ground motion by means of the sample standard deviation over a seismic input population composed of 200 three-component ground motion records collected in a station on seismic bedrock in the same geographical area. We used the evaluated standard deviation to estimate the effect size of the adoption of models with a heterogeneous structure instead of a homogeneous one.

The obtained results permit to highlight the frequency dependence of topography-related amplification, which is controlled by topographic features with characteristic sizes comparable with the associated wavelength. For the homogeneous model, we found a good linear correlation between the plane wave amplification function and the topography's convexity, following the approach introduced by Maufroy et al. (2015). The maximum amplification values in a homogeneous model are however moderate (they do not exceed a factor of 2), but the introduction of the heterogeneous structure causes an aggravation, with modulations that follow the topographic and geological features. In the intermediate and high frequency bands, the aggravation is significant at some spots, in particular in the flat area occupied by the small alluvial basin, where the amplification equals and even exceeds the topography-related amplification at topographic highs. The correlation between amplification and curvature weakens for frequencies above $2 \mathrm{~Hz}$, as soon as the weathering layer is considered in the structure, suggesting caution in the adoption of DEM-based proxies for site amplification in that frequency range. The directional analysis of the amplification in the homogeneous model show frequency dependence, as well. A visual analysis of the polarization level and angle distribution over the area, reveals that on the topographic reliefs, where the polarization is associtated with convexity-related amplification, the polarization tends to be orthogonal to the topography major axis in the low frequency range, whereas in the high frequency range, the polarization changes and tends to be orthogonal to secondary ridges axes. The same analysis reveals that many points on the map are characterized by a polarized response that does not imply amplification. Actually we found that the polarization level is generally uncorrelated with the amplification. We conclude that even if the topography-induced amplification is associated with polarization, the polarization itself does not necessarily indicate amplification, since it could be associated with concavity-related de-amplification. A heterogeneous substratum seems to either attenuate the topography-related polarization effects or produce topographyunrelated amplification and polarization. 
Finally, we analyzed the possibility of topography-induced torsional motions in terms of the scaling factor between the induced rotation rate and the seismic input acceleration. From simple geometrical considerations we expected a linear proportionality between the scaling factor and the tangent of the topographic slope, which was confirmed through a linear regression of the results concerning the homogeneous model case. On the other hand the introduction of the weathering layer clearly implies an aggravation of the induced torsional motion, especially in the high-frequency band. The torsional motion induced by the concurrence of slope and weathering appears to be of the same order of magnitude as that induced by the structural heterogeneity on the basin edge. This finding suggests that induced torsional motion could concur in the aggravation of earthquake effects on rocky hills like the one on which Arquata del Tronto rests.

For both the translational and torsional components, the most evident aggravation of topography-related effects in respect to the homogeneous model is given by the introduction of the weathering layer in the frequency band 4-8 Hz. On the other hand, the introduction of the 3D structure below the weathering layer implies a median aggravation that exceeds the one due to the introduction of the weathering layer in the band 1-4 Hz, but it appears relatively less significant if measured in respect to the variability of the 3D seismic response related to the components' cross-coupling.

\section{Declarations}

\section{Funding}

The research reported in this work was supported by OGS and CINECA under HPC-TRES program award number 2017-22 (J. B.).

\section{Conflicts of interest/Competing interests}

The authors declare no competing interests.

\section{Availability of data and material}

The topography data used in the present study is the $10 \mathrm{~m}$ resolution DEM Tinitaly available at http://tinitaly.pi.ingv.it/. Other material is available on request from the authors.

\section{Code availability}

683

The SPECFEM3D code is an open source software available on the Computational Infrastructure for Geodynamics (http://geodynamics.org). The 3D geological model was computed using the commercial software GeoModeller (https://www.intrepid-geophysics.com/product/geomodeller). Data processing and figures were done using MATLAB (http://www.mathworks.com/products/matlab/) and GMT (https://www.generic-mappingtools.org/).

\section{Authors' contributions}

All authors contributed to the study conception and design. Julie Baron performed the numerical simulations and data processing, Ilaria Primofiore edited the 3D geological models, Peter Klin provided the method description while Giovanna Vessia and Giovanna Laurenzano merged the parts and commented the results. All authors read and approved the final manuscript.

\section{References}

Amanti M, Muraro C, Roma M, et al (2020) Geological and geotechnical models definition for 3rd level seismic microzonation studies in Central Italy. Bull Earthq Eng 18:5441-5473. https://doi.org/10.1007/s10518020-00843-x 
Anagnostopoulos SA, Kyrkos MT, Stathopoulos KG (2015) Earthquake induced torsion in buildings: critical review and state of the art. Earthq Struct 8:305-377. https://doi.org/10.12989/eas.2015.8.2.305

Ashford SA, Sitar N, Lysmer J, Deng N (1997) Topographic effects on the seismic response of steep slopes. Bull Seismol Soc Am 87:701-709

Assimaki D, Jeong S (2013) Ground-Motion Observations at Hotel Montana during the M 7.0 2010 Haiti Earthquake: Topography or Soil Amplification? Bull Seismol Soc Am 103:2577-2590. https://doi.org/10.1785/0120120242

Burjánek J, Edwards B, Fäh D (2014) Empirical evidence of local seismic effects at sites with pronounced topography: a systematic approach. Geophys J Int 197:608-619. https://doi.org/10.1093/gji/ggu014

Calcagno P, Chilès JP, Courrioux G, Guillen A (2008) Geological modelling from field data and geological knowledge: Part I. Modelling method coupling 3D potential-field interpolation and geological rules. Phys Earth Planet Inter 171:147-157. https://doi.org/10.1016/j.pepi.2008.06.013

Castellani A, Stupazzini M, Guidotti R (2012) Free-field rotations during earthquakes: Relevance on buildings. Earthq Eng Struct Dyn 41:875-891. https://doi.org/10.1002/eqe.1163

Conrad O, Bechtel B, Bock M, et al (2015) System for Automated Geoscientific Analyses (SAGA) v. 2.1.4. Geosci Model Dev 8:1991-2007. https://doi.org/10.5194/gmd-8-1991-2015

Cruz-Atienza VM, Tago J, Sanabria-Gómez JD, et al (2016) Long Duration of Ground Motion in the Paradigmatic Valley of Mexico. Sci Rep 6:38807. https://doi.org/10.1038/srep38807

Evans JR, International Working Group on Rotational Seismology (2009) Suggested Notation Conventions for Rotational Seismology. Bull Seismol Soc Am 99:1073-1075. https://doi.org/10.1785/0120080060

Fehr M, Kremers S, Fritschen R (2019) Characterisation of seismic site effects influenced by near-surface structures using 3D waveform modelling. J Seismol 23:373-392. https://doi.org/10.1007/s10950-01809811-0

Galli P, Peronace E, Tertulliani A (2016) Rapporto sugli effetti macrosismici del terremoto del 24 Agosto 2016 di Amatrice in scala MCS. Zenodo

Geli L, Bard P-Y, Jullien B (1988) The effect of topography on earthquake ground motion: A review and new results. Bull Seismol Soc Am 78:42-63

Giallini S, Pizzi A, Pagliaroli A, et al (2020) Evaluation of complex site effects through experimental methods and numerical modelling: The case history of Arquata del Tronto, central Italy. Eng Geol 272:105646. https://doi.org/10.1016/j.enggeo.2020.105646

Graizer V (2009) Low-velocity zone and topography as a source of site amplification effect on Tarzana hill, California. Soil Dyn Earthq Eng 29:324-332. https://doi.org/10.1016/j.soildyn.2008.03.005

Grelle G, Wood C, Bonito L, et al (2018) A reliable computerized litho-morphometric model for development of 3D maps of Topographic Aggravation Factor (TAF): the cases of East Mountain (Utah, USA) and Port au Prince (Haiti). Bull Earthq Eng 16:1725-1750. https://doi.org/10.1007/s10518-017-0272-x

Guidotti R, Castellani A, Stupazzini M (2018) Near-Field Earthquake Strong Ground Motion Rotations and Their Relevance on Tall Buildings. Bull Seismol Soc Am 108:1171-1184. https://doi.org/10.1785/0120170140

Hailemikael S, Lenti L, Martino S, et al (2016) Ground-motion amplification at the Colle di Roio ridge, central Italy: a combined effect of stratigraphy and topography. Geophys J Int 206:1-18. https://doi.org/10.1093/gji/ggw120 
ISPRA (2018) Relazione Illustrativa dello Studio di Microzonazione Sismica propedeutico al Livello 3 della Macroarea 1 Arquata del Tronto e Montegallo (AP) e Allegati (in italian). Rome

Klin P, Laurenzano G, Romano MA, et al (2019) ER3D: a structural and geophysical 3-D model of central Emilia-Romagna (northern Italy) for numerical simulation of earthquake ground motion. Solid Earth 10:931-949. https://doi.org/10.5194/se-10-931-2019

Lanzo G, Tommasi P, Ausilio E, et al (2019) Reconnaissance of geotechnical aspects of the 2016 Central Italy earthquakes. Bull Earthq Eng 17:5495-5532. https://doi.org/10.1007/s10518-018-0350-8

Laurenzano G, Barnaba C, Romano MA, et al (2019) The Central Italy 2016-2017 seismic sequence: site response analysis based on seismological data in the Arquata del Tronto-Montegallo municipalities. Bull Earthq Eng 17:5449-5469. https://doi.org/10.1007/s10518-018-0355-3

Lee VW, Trifunac MD (1985) Torsional accelerograms. Int J Soil Dyn Earthq Eng 4:132-139. https://doi.org/10.1016/0261-7277(85)90007-5

Lee WHK, Çelebi M, Todorovska MI, Igel H (2009) Introduction to the Special Issue on Rotational Seismology and Engineering Applications. Bull Seismol Soc Am 99:945-957. https://doi.org/10.1785/0120080344

Luo Y, Fan X, Huang R, et al (2020) Topographic and near-surface stratigraphic amplification of the seismic response of a mountain slope revealed by field monitoring and numerical simulations. Eng Geol 271:105607. https://doi.org/10.1016/j.enggeo.2020.105607

Massa M, Barani S, Lovati S (2014) Overview of topographic effects based on experimental observations: meaning, causes and possible interpretations. Geophys J Int 197:1537-1550. https://doi.org/10.1093/gji/ggt341

Maufroy E, Cruz-Atienza VM, Cotton F, Gaffet S (2015) Frequency-Scaled Curvature as a Proxy for Topographic Site-Effect Amplification and Ground-Motion Variability. Bull Seismol Soc Am 105:354-367. https://doi.org/10.1785/0120140089

Maufroy E, Cruz-Atienza VM, Gaffet S (2012) A Robust Method for Assessing 3-D Topographic Site Effects: A Case Study at the LSBB Underground Laboratory, France. Earthq Spectra 28:1097-1115. https://doi.org/10.1193/1.4000050

Moore ID, Grayson RB, Ladson AR (1991) Digital terrain modelling: A review of hydrological, geomorphological, and biological applications. Hydrol Process 5:3-30. https://doi.org/10.1002/hyp.3360050103

Newmark N (1969) Torsion in symmetrical buildings. In: Proceedings of the 4th World Conference Earthq. Eng. ASCE

Paolucci R (2002) Amplification of earthquake ground motion by steep topographic irregularities. Earthq Eng Struct Dyn 31:1831-1853. https://doi.org/10.1002/eqe.192

Paolucci R (1999) Numerical evaluation of the effect of cross-coupling of different components of ground motion in site response analyses. Bull Seismol Soc Am 89:877-887

Paolucci R, Mazzieri I, Smerzini C (2015) Anatomy of strong ground motion: near-source records and threedimensional physics-based numerical simulations of the Mw 6.0 2012 May 29 Po Plain earthquake, Italy. Geophys J Int 203:2001-2020. https://doi.org/10.1093/gji/ggv405

Pedersen H, Le Brun B, Hatzfeld D, et al (1994) Ground-motion amplitude across ridges. Bull Seismol Soc Am $84: 1786-1800$

Peter D, Komatitsch D, Luo Y, et al (2011) Forward and adjoint simulations of seismic wave propagation on fully unstructured hexahedral meshes. Geophys J Int 186:721-739. https://doi.org/10.1111/j.1365246X.2011.05044.x 
Pischiutta M, Cianfarra P, Salvini F, et al (2018) A systematic analysis of directional site effects at stations of the Italian seismic network to test the role of local topography. Geophys J Int 214:635-650. https://doi.org/10.1093/gji/ggy133

Primofiore I, Baron J, Klin P, et al (2020) 3D numerical modelling for interpreting topographic effects in rocky hills for Seismic Microzonation: The case study of Arquata del Tronto hamlet. Eng Geol 279:105868. https://doi.org/10.1016/j.enggeo.2020.105868

Puglia R, Vona M, Klin P, et al (2013) Analysis of Site Response and Building Damage Distribution Induced by the 31 October 2002 Earthquake at San Giuliano di Puglia (Italy). Earthq Spectra 29:497-526. https://doi.org/10.1193/1.4000134

Puzzilli LM, Ferri F, Eulilli V, et al (2019) Integrated geophysical methods for the seismic site characterization of Arquata del Tronto. Roma, Italy

Savage B, Komatitsch D, Tromp J (2010) Effects of 3D Attenuation on Seismic Wave Amplitude and Phase Measurements. Bull Seismol Soc Am 100:1241-1251. https://doi.org/10.1785/0120090263

Seriani G, Priolo E (1994) Spectral element method for acoustic wave simulation in heterogeneous media. Finite Elem Anal Des 16:337-348. https://doi.org/10.1016/0168-874X(94)90076-0

Spudich P, Fletcher JB (2008) Observation and Prediction of Dynamic Ground Strains, Tilts, and Torsions Caused by the Mw 6.0 2004 Parkfield, California, Earthquake and Aftershocks, Derived from UPSAR Array Observations. Bull Seismol Soc Am 98:1898-1914. https://doi.org/10.1785/0120070157

Stupazzini M, de la Puente J, Smerzini C, et al (2009) Study of Rotational Ground Motion in the Near-Field Region. Bull Seismol Soc Am 99:1271-1286. https://doi.org/10.1785/0120080153

Tarquini S, Isola I, Favalli M, et al (2007) TINITALY/01: a new Triangular Irregular Network of Italy. Ann Geophys 50:407-425. https://doi.org/10.4401/ag-4424

Wood CM, Cox BR (2016) Comparison of Field Data Processing Methods for the Evaluation of Topographic Effects. Earthq Spectra 32:2127-2147. https://doi.org/10.1193/111515EQS170M

Xie Z, Komatitsch D, Martin R, Matzen R (2014) Improved forward wave propagation and adjoint-based sensitivity kernel calculations using a numerically stable finite-element PML. Geophys J Int 198:17141747. https://doi.org/10.1093/gji/ggu219

Zevenbergen LW, Thorne CR (1987) Quantitative analysis of land surface topography. Earth Surf Process Landf 12:47-56. https://doi.org/10.1002/esp.3290120107

Zhou H, Li J, Chen X (2020) Establishment of a seismic topographic effect prediction model in the Lushan Ms 7.0 earthquake area. Geophys J Int 221:273-288. https://doi.org/10.1093/gji/ggaa003 


\section{Figures}
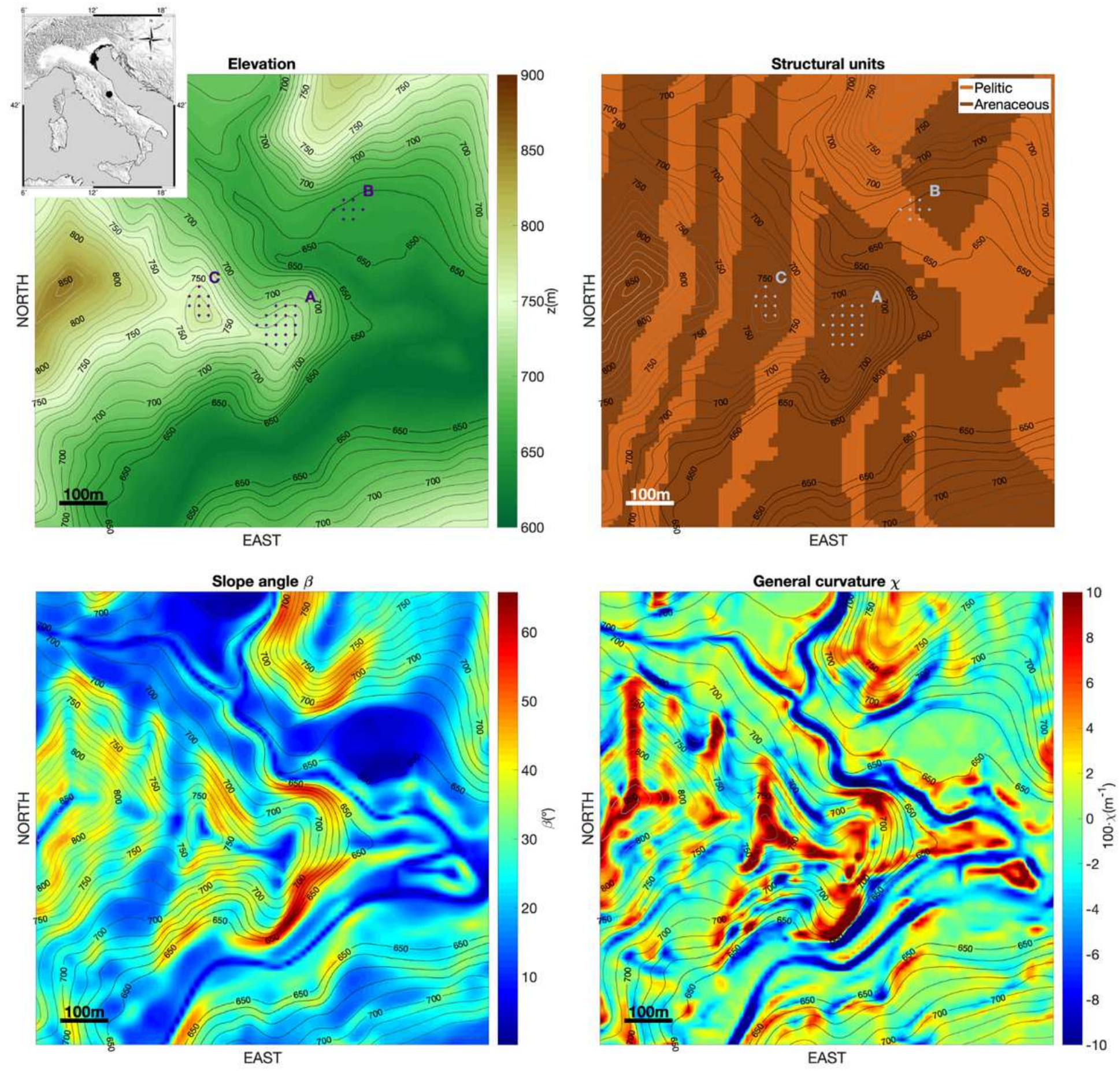

\section{Figure 1}

topographic and morphologic characterization of the studied area. The location of the area in the Italian peninsula is evidenced with the black dot in the small map overlapping the elevation map (upper left). The cross marks in the elevation map indicate the virtual seismic stations in three sites of interest: site $A$ corresponds to Arquata del Tronto hamlet, site B to Borgo in the valley and Site $\mathrm{C}$ to the Castle promontory. In the structural model map (upper right) we depict the alternance of pelitic and arenaceous 
formations just below the weathering layer. The topographic slope and curvature are shown in the bottom left and right maps respectively

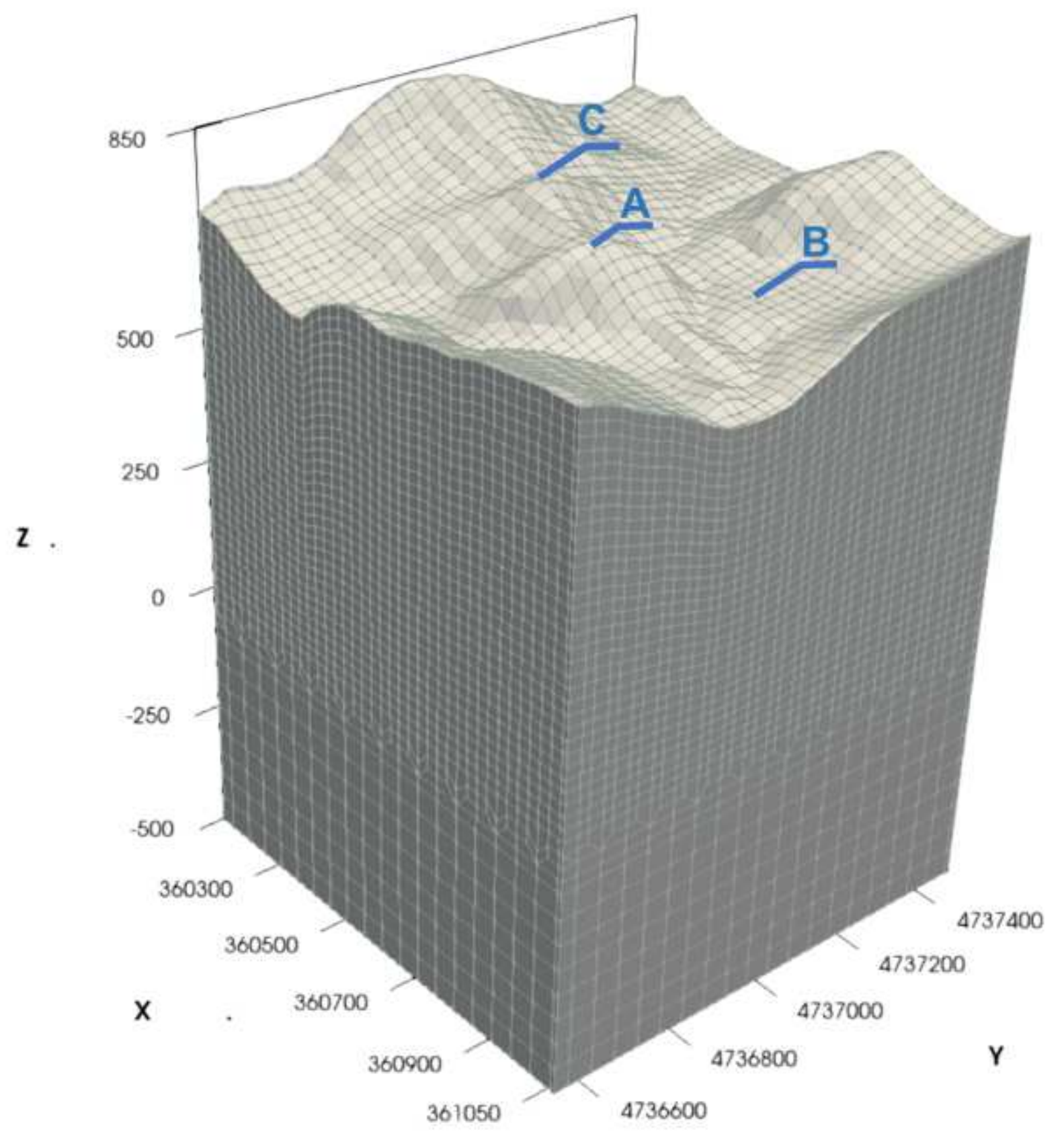

\section{Figure 2}

the central portion of the numerical mesh used for numerical simulations. The locations of the sites $A, B$ and $\mathrm{C}$ are shown. Length units are in meters, $\mathrm{x}$-direction is eastward 


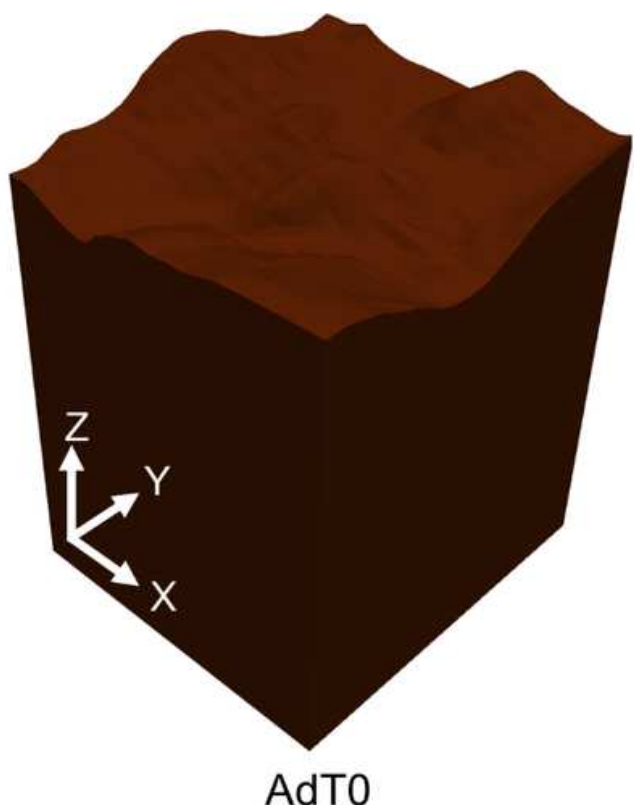

AdTO

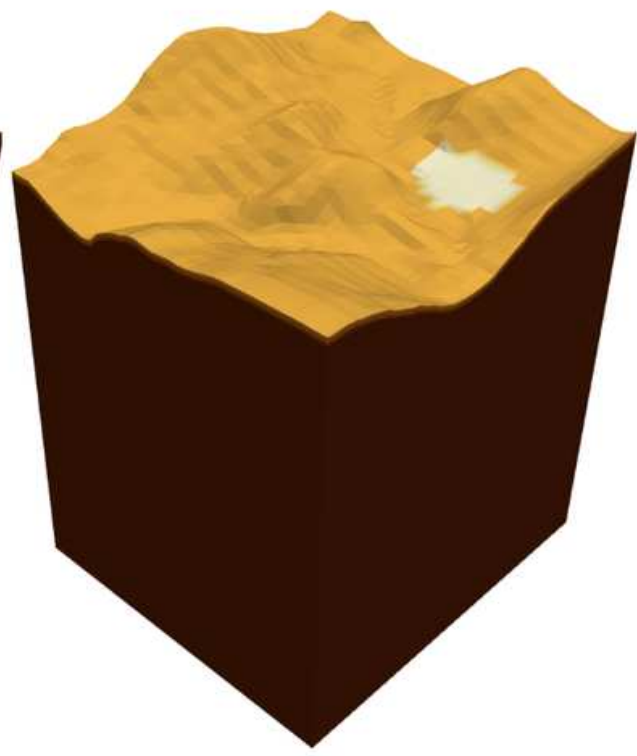

AdT1

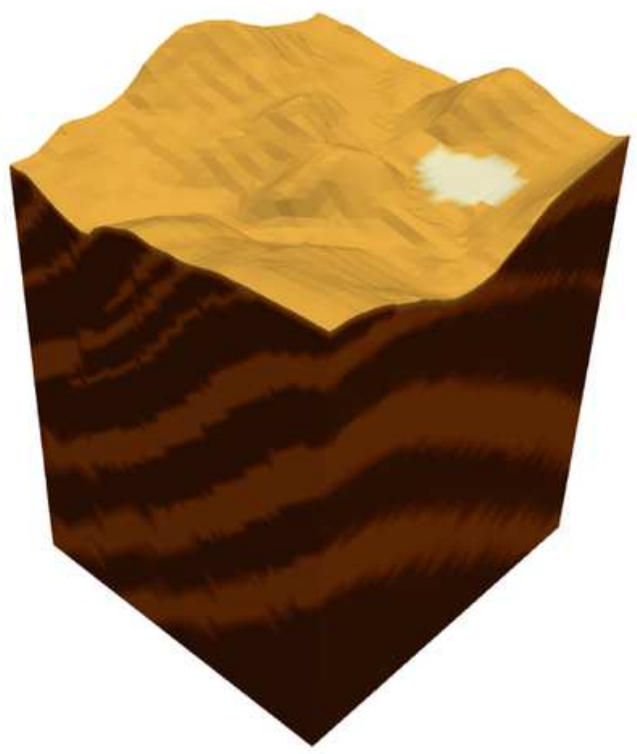

AdT2

\section{Figure 3}

representation of the three input models clipped on the $1 \mathrm{~km} 2$ square area of interest. AdT0 is the homogeneous model, AdT1 is the homogeneous model with a weathering layer and an alluvial, AdT2 is the full 3D model of Arquata del Tronto retrieved from Primofiore et al. (2020) 

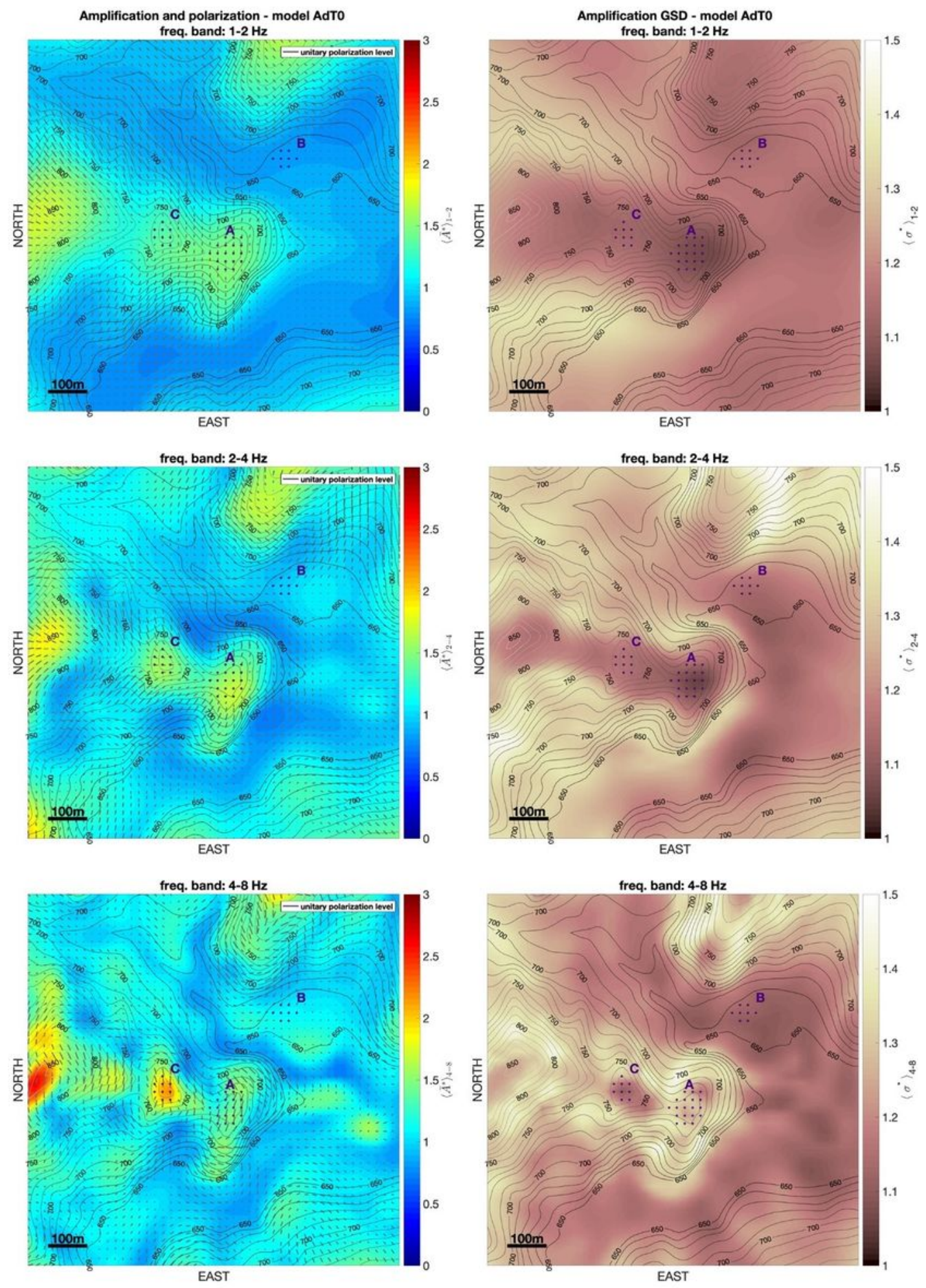

\section{Figure 4}

left) distribution of horizontal plane-wave amplification and polarization computed for the homogeneous model in the three frequency bands $1-2 \mathrm{~Hz}, 2-4 \mathrm{~Hz}$ and $4-8 \mathrm{~Hz}$. Grey headless arrows in the maps in the left column indicate the direction of polarization. The polarization level is indicated by the arrow length; right) the geometrical standard deviation corresponding to the amplification values on the left 

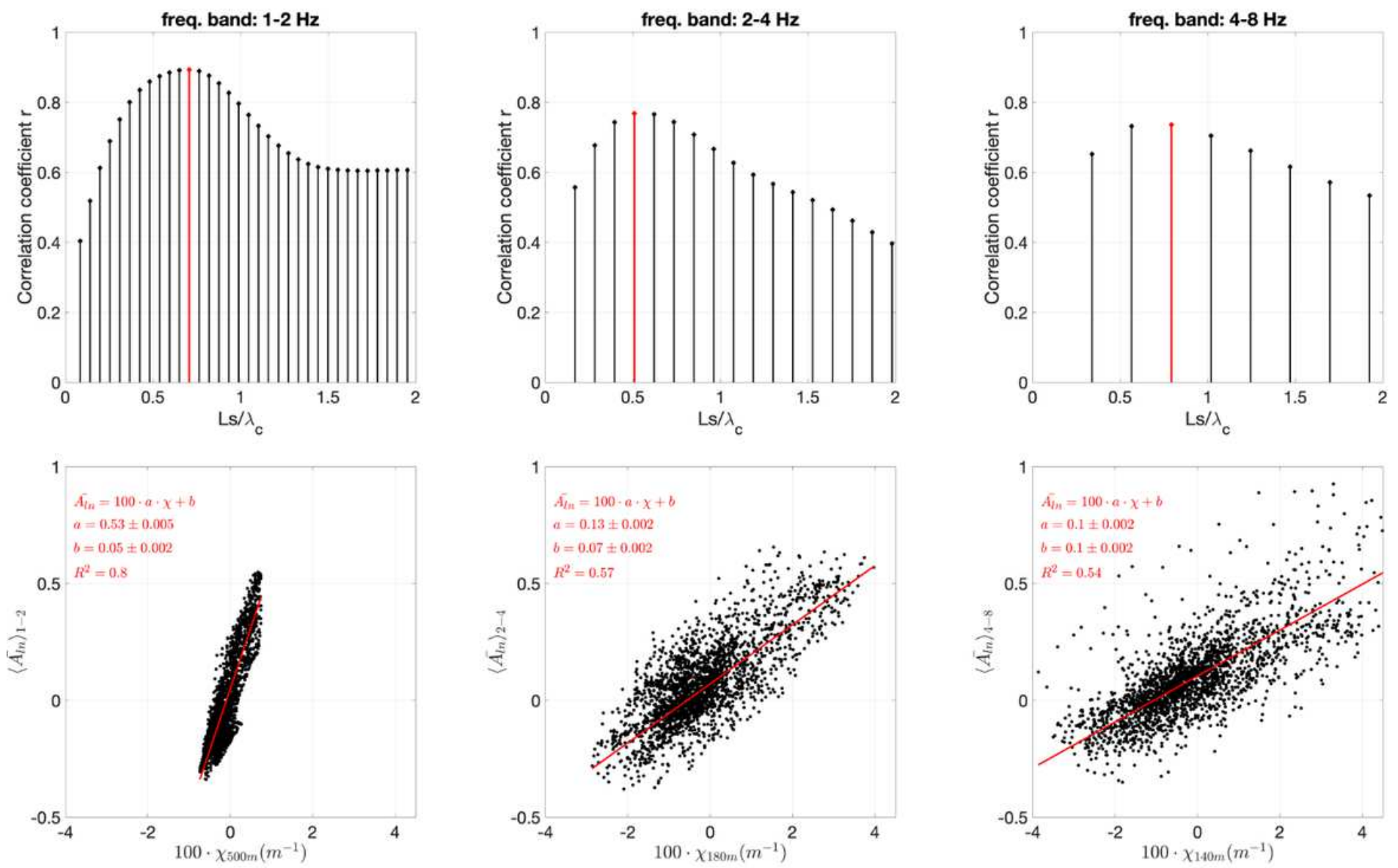

Figure 5

Please see the Manuscript PDF file for the complete figure caption.
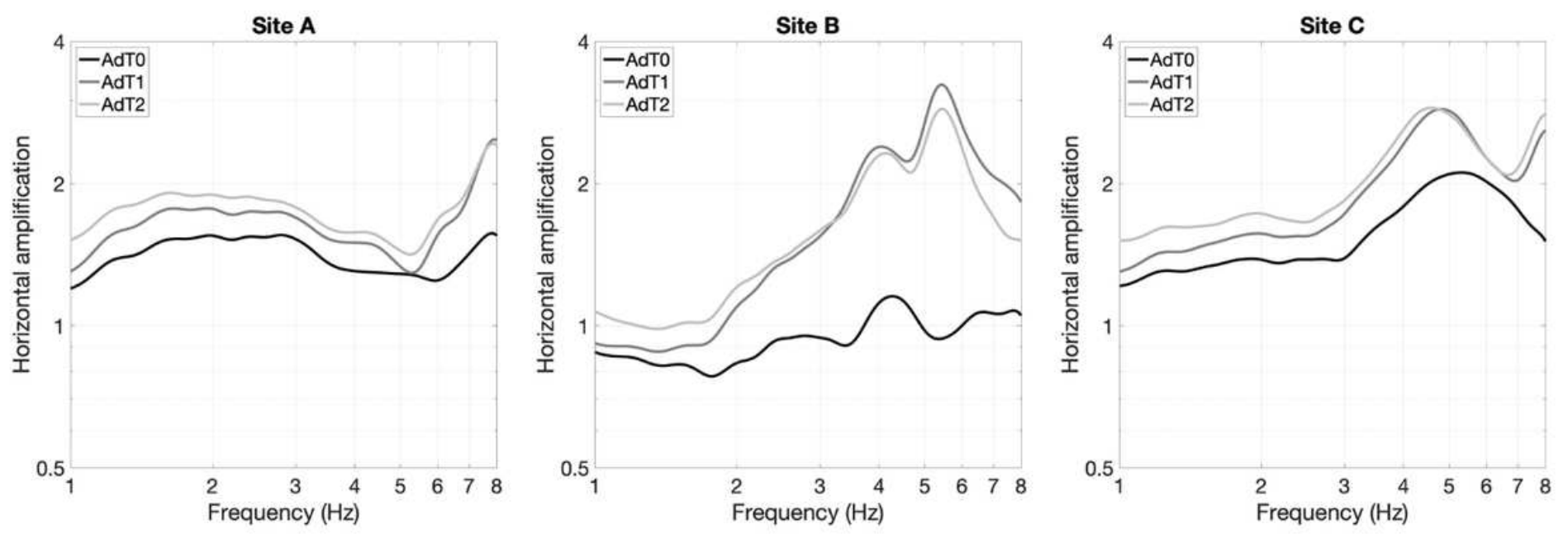

Figure 6

average horizontal plane-wave amplification at Site A (Arquata center), Site B (Borgo) and Site C (Arquata castle) for homogeneous AdT0 model (black), inhomogeneous AdT1 model (dark grey) and 3D model 
AdT2 (light grey). The average implies the stations covering each site, indicated as crossmarks in the maps in Fig.4
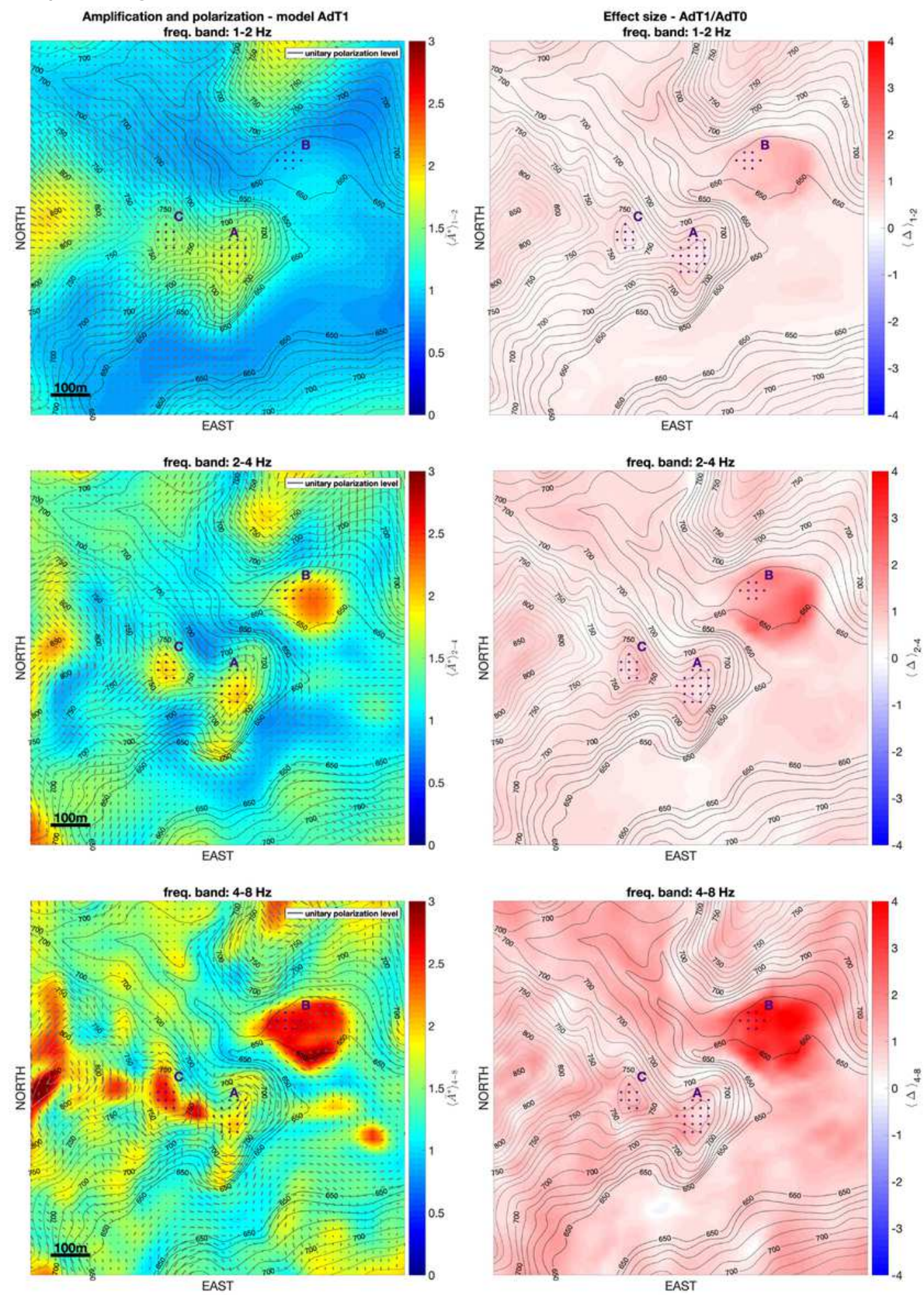

Figure 7

left) horizontal plane-wave amplification distribution pattern at Arquata del Tronto computed for the model AdT1 and in the frequency bands $1-2 \mathrm{~Hz}, 2-4 \mathrm{~Hz}$ and $4-8 \mathrm{~Hz}$. Same notation as in Fig.4; right) the 
effect size (see Eq. 17 in the text) in the amplification values for the model AdT1 in respect to model AdTO
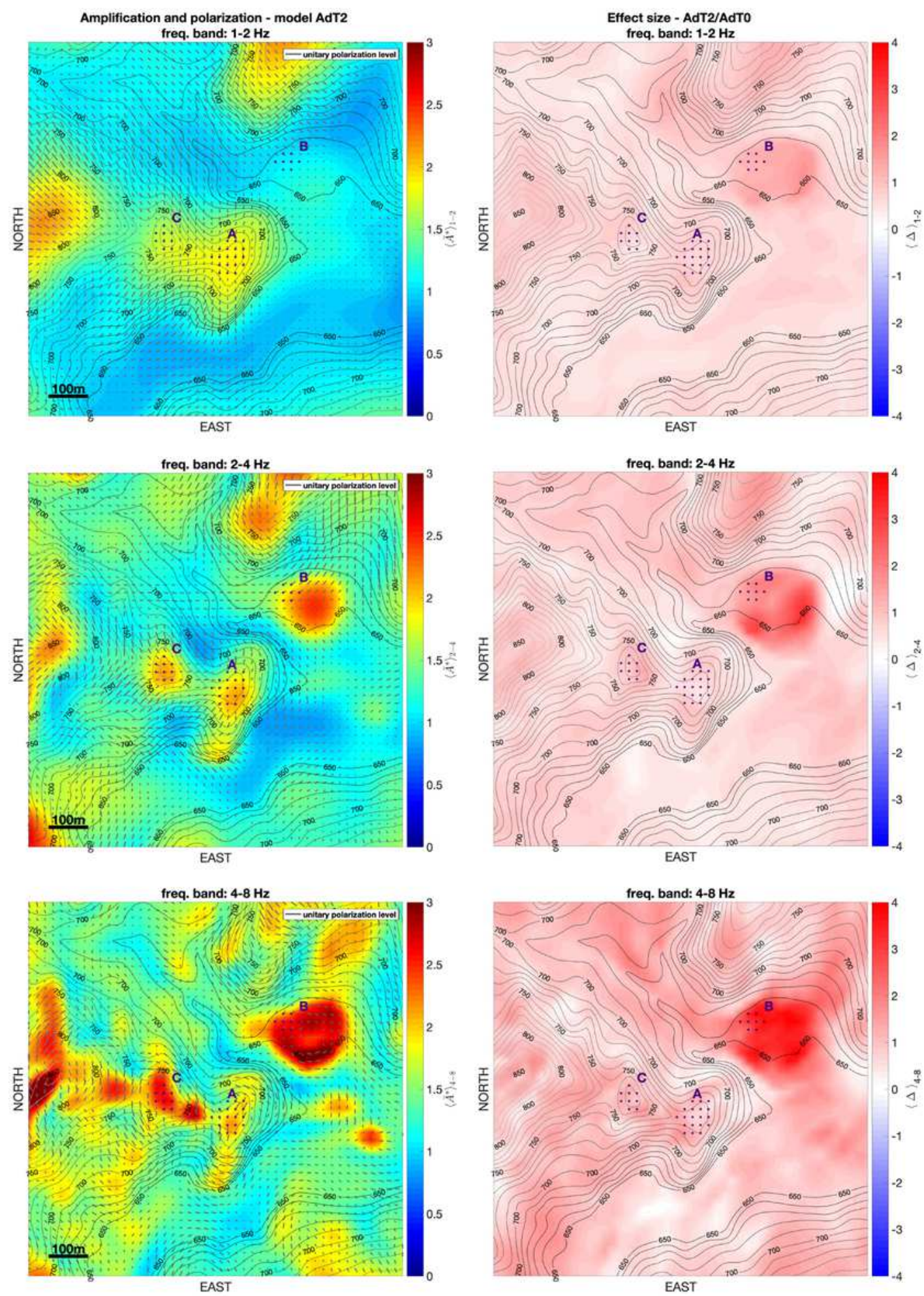

Figure 8

left) horizontal plane-wave amplification distribution pattern at Arquata del Tronto computed for the model AdT2 and in the frequency bands $1-2 \mathrm{~Hz}, 2-4 \mathrm{~Hz}$ and $4-8 \mathrm{~Hz}$. Same notation as in Fig.4; right) the 
effect size (see Eq. 17 in the text) in the amplification values for the model AdT2 in respect to model AdTO
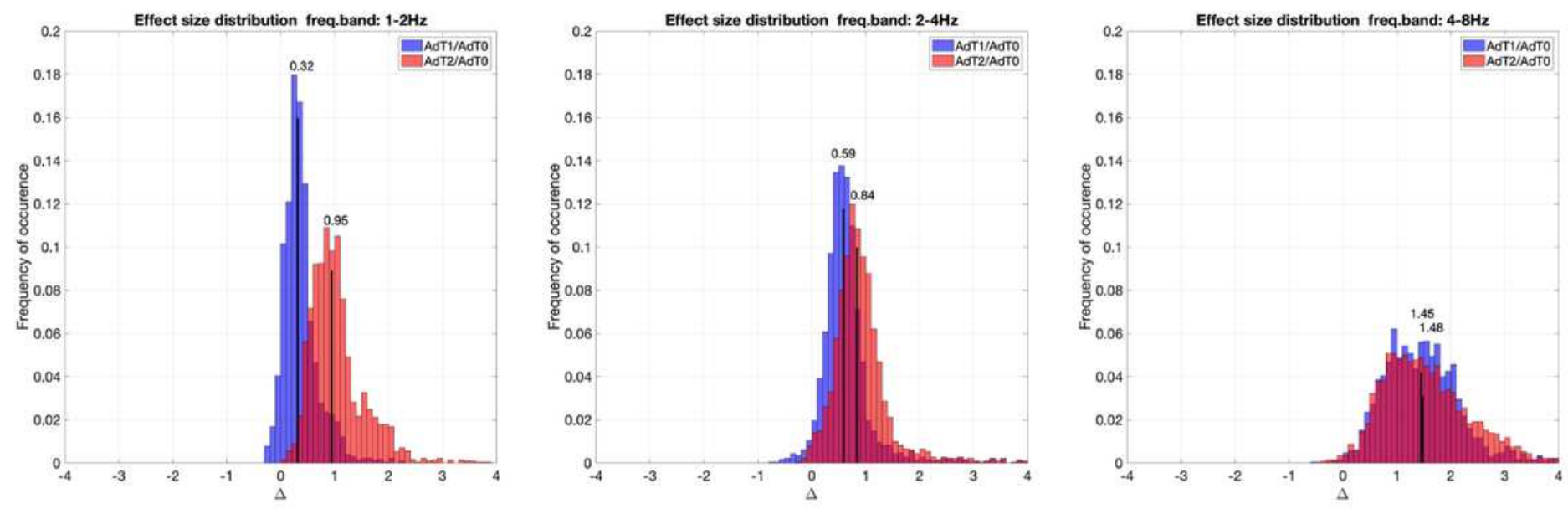

\section{Figure 9}

histograms of the amplification effect size values over the grid points sampling the studied area for the frequency bands: $1-2 \mathrm{~Hz}, 2-4 \mathrm{~Hz}$ and $4-8 \mathrm{~Hz}$. The median value is indicated in order to assess the overall differences between the changes in the amplification functions due to the variations in the structural models

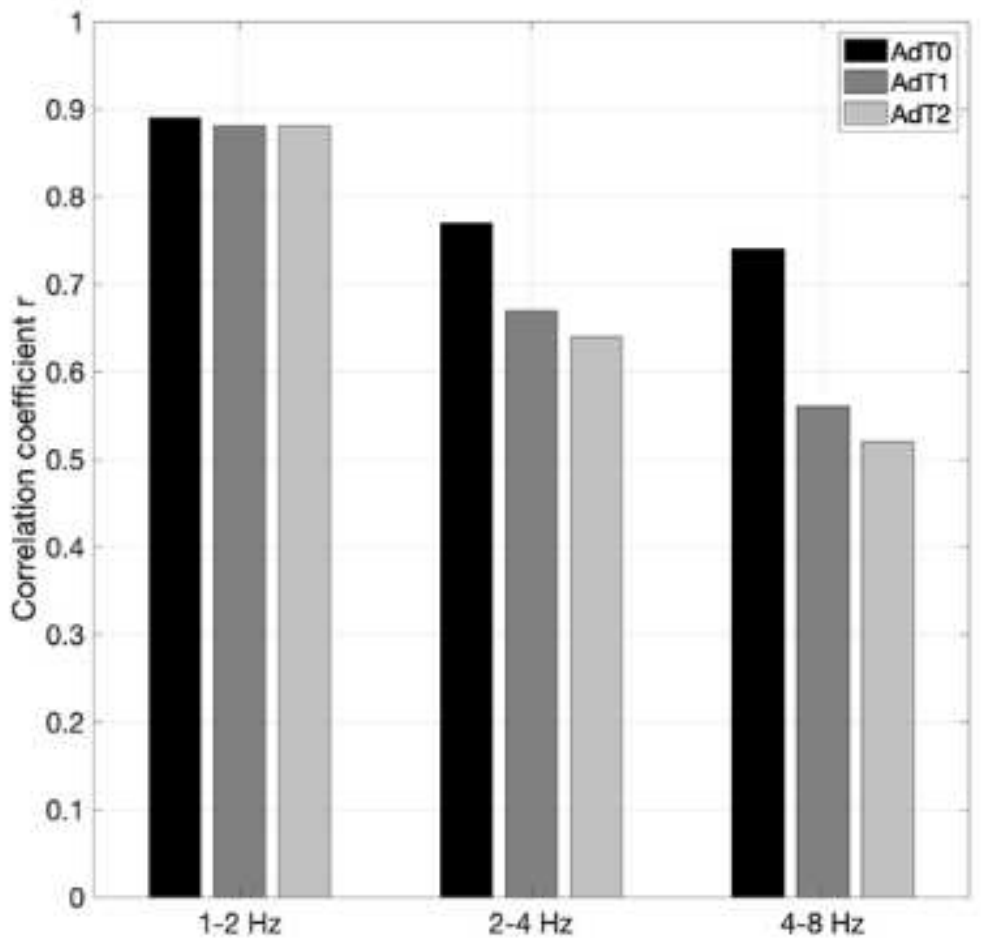

Figure 10 
correlation coefficients between averaged horizontal plane-wave amplification and smoothed curvature obtained in the three frequency bands of interest for homogeneous model AdT0 (black), inhomogeneous model AdT1 (dark grey) and 3D model AdT2 (light grey)
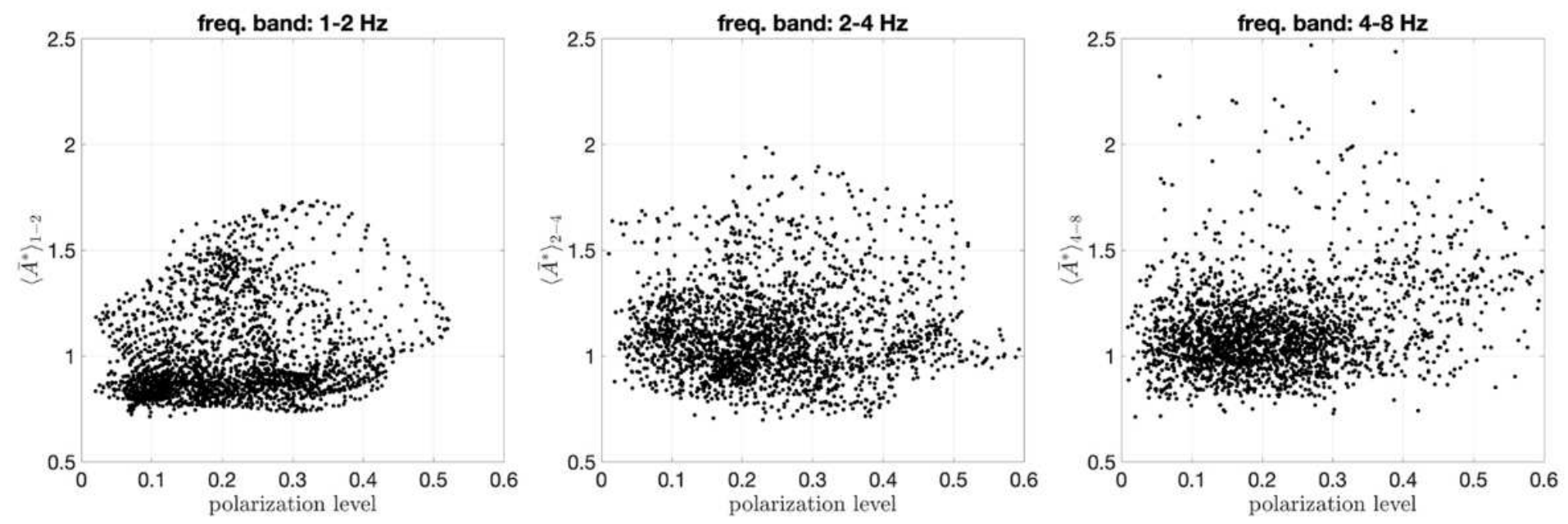

Figure 11

distribution of amplification values in function of the polarization level in the three frequency bands for the Model AdT0 case 


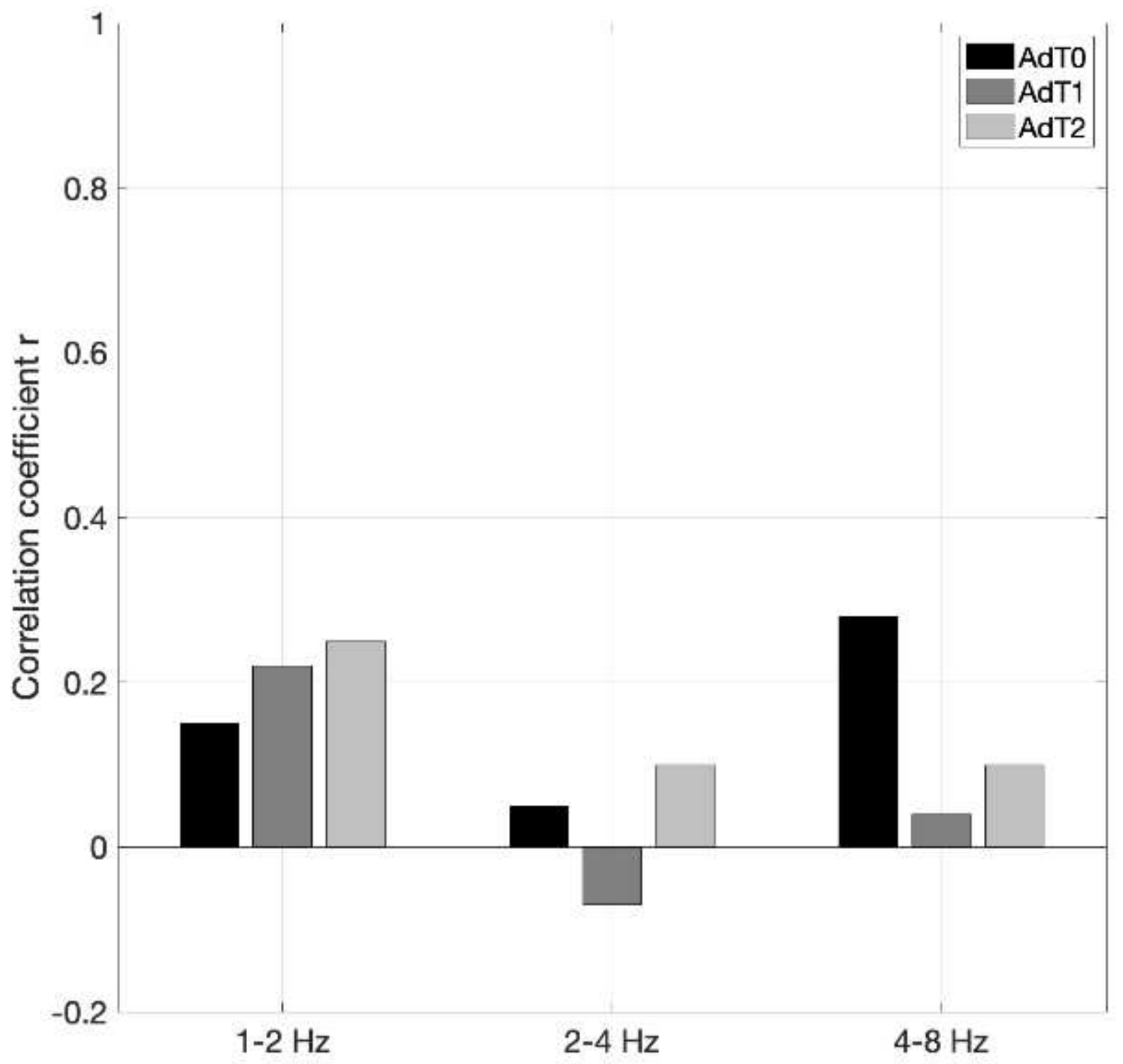

Figure 12

the values of the linear correlation coefficient between the polarization level and amplification 

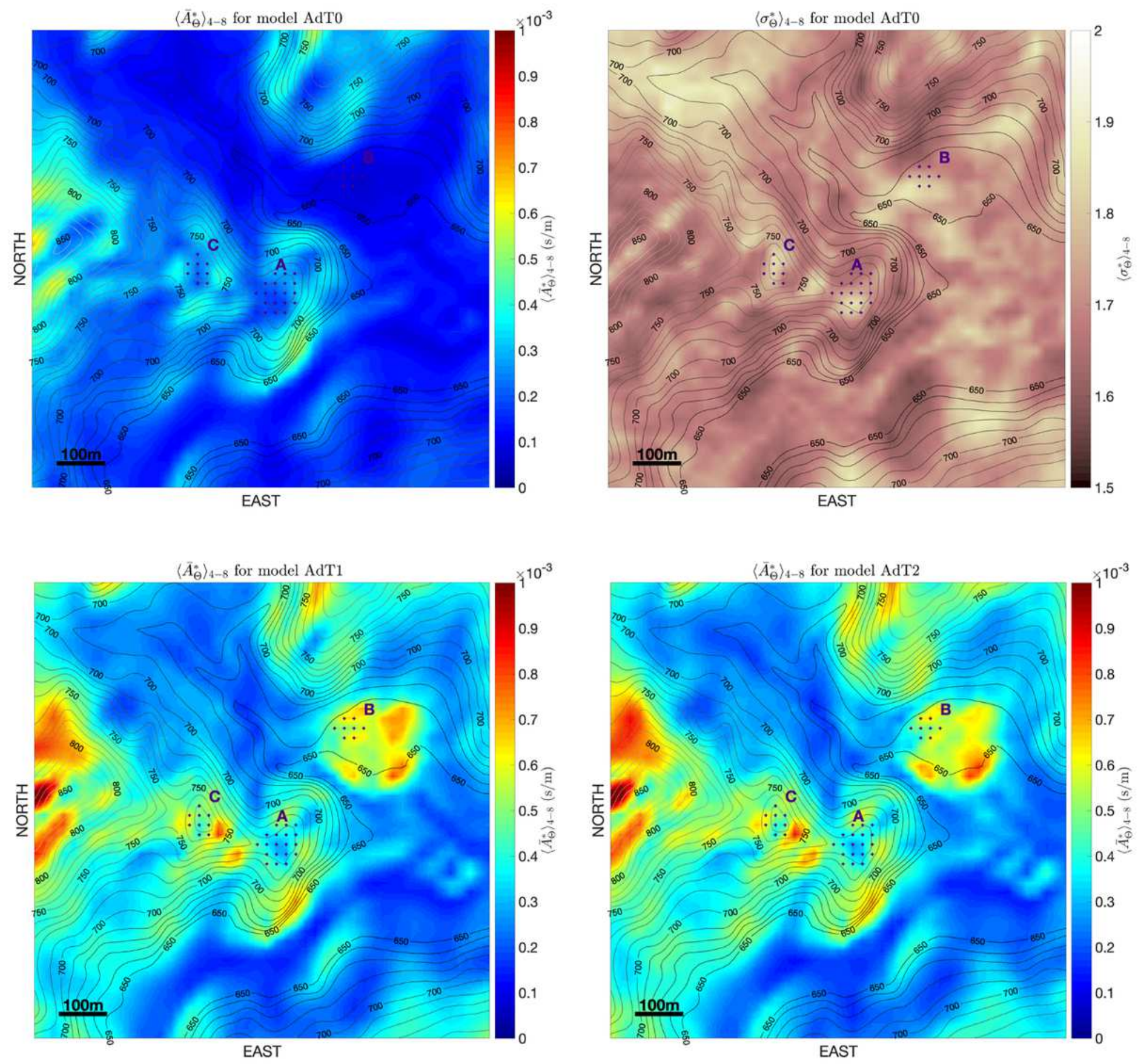

\section{Figure 13}

Please see the Manuscript PDF file for the complete figure caption. 

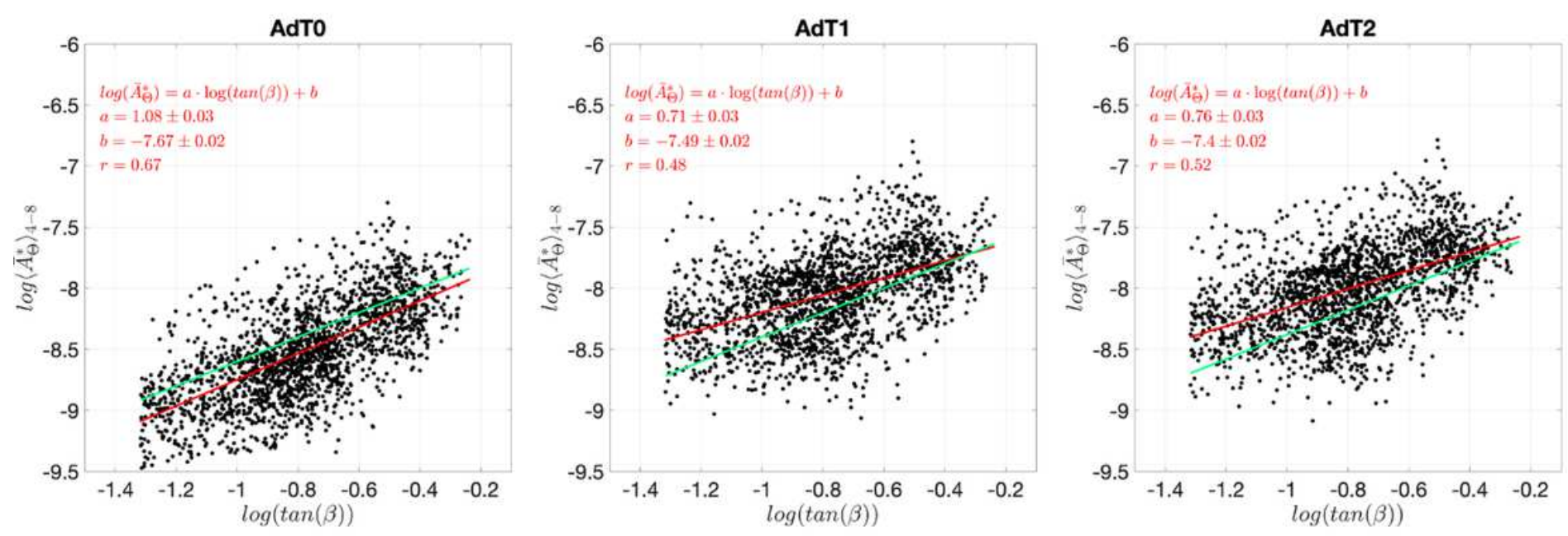

Figure 14

Please see the Manuscript PDF file for the complete figure caption.
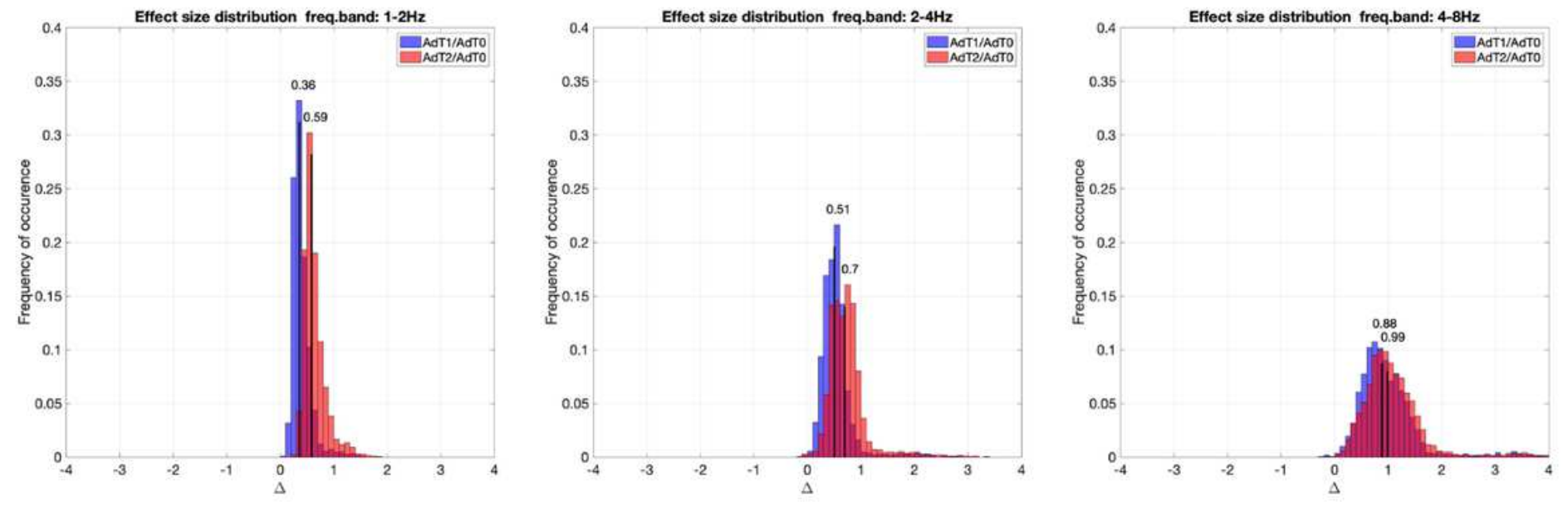

Figure 15

Please see the Manuscript PDF file for the complete figure caption. 